Aus dem Pathologischen Institut zu Freiburg i. Br. (Geheimrat Prof. Dr. Aschoff) und dem Pathologischen Institut zu Dortmund (Prof. Dr. Schridde).

\title{
Zellreiche Myome und Myosarkome des Uterus.
}

Von

Heinrieh Raab.

(Hierzu Tafel XI und 8 Textfiguren.)

Die Frage nach der Häufigkeit des Auftretens von Sarkom in Uterusmyomen findet neuerdings wieder ein praktisches Interesse, seitdem die Röntgenbestrahlung in die Therapie der Uterusmyome eingeführt ist.

Zuletzt wurde auf der Frankfurter Versammlung der Mittelrheinischen Gesellschaft für Geburtshülfe und Gynäkologie über das Thema verhandelt, und dabei wurden sehr verschiedene Anschauungen über die Häufigkeit des Vorkommens von Sarkom in Myomen geänssert. Gauss wies auf die Schwierigkeit der pathologisch - anatomischen Diagnose des Myosarkoms hin und glaubt darin eine Erklärung für die auseinandergehenden Meinungen zu finden, insofern dass vielleicht häufig bei der Aufstellung von Statistiken zellreiche, aber benigne Myome als Myosarkome diagnostiziert wurden.

Zur Klärung der Frage habe ich auf Veranlassung von Herrn Prof. Schridde eine Anzahl von Präparaten einer genaueren histologischen Untersuchung unterzogen. Mein Material bestand aus vier Fällen von Uterusgeschwülsten, die in dem Freiburger Pathologischen Institut in den Jahren 1910 und 1911 beobachtet und bei der damaligen Untersuchung folgendermassen beurteilt worden waren. Bei zwei Geschwülsten lautete die Diagnose Myosarkom, bei einer Hyom mit sarkomatösen Partien, bei der letzten „schnellwachsendes Myom, aber noch nicht Myosarkom". In den vorausgegangenen Jahren hatten sich unter dem Material des Instituts keine derartigen Fälle feststellen lassen. 
Aschoff unterscheidet in seinem Lehrbuch (1911) metastasierende Myome von histologisch gutartigem Aufbau, "welche durch gelegentlichen Einbruch in Gefässe bezw. durch besondere Widerstandskraft in der Blutbahn in den Lungen zur Ansiedlung und zum Wachstum gelangen", und hält es für eine untergeordnete Streitfrage, ob man diese Geschwülste als maligne Leiomyome bezeichnen will, da zwischen Gutartigkeit und Bösartigkeit fliessende Uebergänge bestehen. Ferner sarkomatöse Geschwülste, die schon im Primärtumor infiltrierendes Wachstum zeigen. Diese Geschwülste haben eine weichere Konsistenz und weisen makroskopisch ein markiges, mehr weissliches Aussehen auf. Mikroskopisch bestehen sie aus Spindelzellen, die bald gleichartige, jungen Muskelzellen ähnliche Fasern, bald eine grosse Variabilität der Zellleiber und. Kerne bezüglich Grösse, Form und Chromatinanordnung zeigen. Häufig sind Zellen mit Riesenkernen und mehrkernige Riesenzellen. Histogenetisch muss für die Geschwülste, deren Zellen Aehnlichkeit mit jungen Muskelzellen zeigen, an eine Abstammung von Myoblasten gedacht werden. Die polymorphzelligen Geschwiilste können abstammen von myogenem Gewebe (Myosarkom) oder von Fibroblasten (Fibrosarkom) oder als echte Mischgeschwülste von beiden Gewebsarten (Myofibrosarkom).

Das Lehrbuch von Kaufmann (1911) führt gleichfalls das Vorkommen von typischen Myomen, die Metastasen setzen, an. Bei der sarkomatösen "Entartung" von Fibromyomen unterscheidet Kaufmann zweierlei. Einmal kommt ein Uebergang des Myomgewebes in dichtgelagerte Sarkomzellen vor, die sich durch breitere und kürzere oder durch vielgestaltete Form der Zellleiber und Kerne von den Muskelzellen unterscheiden. Die Sarkomzellen dieser Geschwülste (Myoma sarkomatodes) sind bösartig wuchernde Muskelzellen, haben also keine "Metaplasie", sondern eine „Entartung" erfahren. Dann erkennt Kaufmann das Vorkommen von Geschwülsten an, bei denen das Sarkom aus dem fibrösen Teil des Myofibroms hervorgegangen ist (Myosarkom ev. Fibro-Myo-Sarkom).

Nach Schmaus-Herxheimer (1910) werden die Uterusmyome in seltenen Fällen maligne und nehmen dann meist eine sarkomatöse Struktur an, die neben typischen Muskelfasern spindelige Zellen zeigt (Myosarkom, Myoblastisches Sarkom). Noch seltener metastasieren einfache Myome.

Sehr eingehend mit den Uterusgeschwülsten, in denen Sarkom und Myom in Beziehung stehen, hat sich neuerdings wiederholt 
Robert Meyer beschäftigt. Nach seiner Auffassung müsste eine Einteilung all dieser Geschwülste nach histologenetischen Gesichtspunkten vorgenommen werden. Eine solche ist indessen heute noch nicht möglich, da über die Histogenese keine Einigkeit erzielt ist, obwohl gerade dieser Frage seit Beginn der Literatur das Interesse der Autoren zugewandt war. Robert Meyer verzichtet deshalb auf die histogenetische Einteilung dieser Geschwülste und betrachtet sie lediglich unter dem Gesichtspunkt der augenblicklichen Morphologie. $\mathrm{Er}$ ist sich dabei wohl bewusst, dass bei längerem Bestehen der Geschwulst diese augenblicklich sichtbare Morphologie durch Ausreifen der Zellen sich noch hätte ändern können. Danach teilt er alle Sarkome ein in muskelzellige und bindegewebszellige. Den Namen Myosarkom will er im Virchow'schen Sinne auf die Tumoren beschränkt wissen, die echte Mischgeschwülste darstellen, in denen also Myom und Sarkom als zwei selbständige Geschwülste ohne genetische Beziehungen nebeneinander vorkommen. Auf die Namen Sarcoma myogenes, fibrogenes, angiogenes oder - für die Annahme einer Entwicklung aus unreifen Keimen - myoblastisches, fibroblastisches, angioblastisches Sarkom glaubt er besser zu verzichten, eben weil die Histogenese nicht in jedem Falle nachzuweisen sei.

Unter den Begriff des muskelzelligen Sarkoms reiht Robert Meyer auch das metastasierende oder maligne Myom ein. Er setzt sich dadurch in bewussten Gegensatz zu den Pathologen, die unter "malignem Myom" einen histologisch gutartigen Tumor verstehen, der nur klinisch durch zufälligen Einbruch in die Blutbahn seine Malignität zeigt. Eine derartige Verschleppung ron Myomzellen hält Meyer für genau so ausgeschlossen wie von Zellen normaler Muskulatur. Dann aber sieht er bei den malignen Myomen auch eine histologische Malignität und zwar in der mangelhaften Ausreifung der Muskelzellen. Diese dokumentiert sich einmal in der ungenügenden Ausbildung von Interzellularsubstanz, Myogliafibrillen, dann aber auch in der Morphologie der Zellen, besonders ihrer Kerne. Nicht die von früheren Autoren bei "metastasierenden Myomen" angegebene schwache Entwicklung des Bindegewebes gibt dem Gewebe die Möglichkeit zu einem schrankenlosen Wuchern (Schlagenhaufer), vielmehr ist der Mangel an Bindegewebe, oder besser gesagt, das Fehlen der Myogliafibrillen bereits der Ausdruck eines gestörten Zellwachstums. Ausgereifte Muskelzellen sondern viel Myogliafibrillen ab, im muskelzelligen Sarkom dagegen findet 
ein überstürztes Zellwachstum statt, die Zellen reifen nicht aus, und es kann deshalb nicht zur genügenden Produktion ron Bindegewebe kommen. Die Zellen des malignen Myoms können anfangs von denen eines gewöhnlichen Myoms nicht unterschieden werden, später zeigen sie die für die "Sarkomzelle" charakteristischen Veränderungen der Gestalt. Diese durch mangelhafte Produktion von Interzellularsubstanz und durch ihre Zellform charakterisierten, nicht ausgereiften Muskelzellen sind fakultativ maligne, das heisst, sie können unter ihnen günstigen Umständen Metastasen setzen. Das maligne Myom ist aber „kein ursprünglich einfaches, sekundär destruierend wachsendes Myom, sondern ein von vorneherein zelldichter, schnellwachsender Tumor, mit der Fähigkeit, Metastasen $\mathrm{zu}$ bilden und zu destruieren".

Robert Meyer hält aus diesen Gründen das maligne Myom für die Vorstufe des muskelzelligen Sarkoms und möchte es schon zu diesen gerechnet wissen. Uebergangsformen kommen vor. So hat er eine fortlaufende Reihe vom "malignen Myom" zum reinen muskelzelligen Sarkom bis zu den Sarkomgeschwülsten mit ausgesprochenster Polymorphie der Zellen aufstellen können. Er nimmt nicht wie frühere Autoren (v. Kahlden, Williams, Pick) eine Metaplasie der Muskelzellen in Bindegewebszellen an, sondern vielmehr eine "Degeneration" von Muskelzellen, deren Sarkomcharakter anfangs dem Auge verborgen ist, später aber in der Form der sogenannten Sarkomzellen erkennbar wird.

Die Bezeichnung dieser Geschwülste als Sarkome hält Meyer, im Gegensatz zu Ribbert, für erlaubt, da der Name Sarkom nicht nur für die heterologen Geschwülste des Bindegewebes im engeren Sinne angewandt ist.

Ueber das morphologische Aussehen der sogenannten Sarkomzellen gibt Robert Meyer an, dass sie Uebergangsformen zwischen Muskelzellen und den ungewöhnlichsten Zellformen darstellen. Oft sind sie von den gewöhnlichen Zellen eines Fibroms oder Myoms nicht zu unterscheiden. Sind Veränderungen bemerkbar, so werden Zellen und Kerne der Spindelzellen grösser. Die Kerne nehmen unregelmässige Formen an, werden runder, sehen wie aufgeblasen aus und zeigen eine wechselnde Färbbarkeit. Dieselben Veränderungen erleiden die Muskelzellen, so dass sie teilweise von Bindegewebszellen nicht zu unterscheiden sind. Die veränderten Muskelzellen zeigen zunehmende Länge der Kerne und eine amitotische Teilung oder einen Zerfall in mehrere Abschnitte. Die Kerne 
lassen eiue "Punktierung" mit einzelnen gröberen Chromatinkörnchen erkennen, während das übrige Kernplasma heller und durchsichtiger wird. Im weiteren Verlauf quellen die Kerne auf, werden gelappt und zerfallen schliesslich. Dieses Sichtbarwerden des schon primär vorhandenen Sarkomcharakters der Zellen erklärt R. Meyer durch eine später eintretende Degeneration der Sarkomzellen infolge eines abnormen Stoffwechsels. Er weist auf den Umstand hin, dass gleichzeitig mit der Veränderung der Kerne eine hyaline oder schleimige Degeneration der Interzellularsubstanz bis zur Nekrose auftritt. Gerade diese proportional dem Grade der sichtbaren Kernveränderungen einhergehenden regressiven Metamorphosen in diesen Geschwülsten stützen ihn in der Auffassung, dass die Kernveränderungen selbst nur ein Zeichen der Zelldegeneration sind.

Nach dem glaubt Robert Meyer bei diesen muskelzelligen Sarkomen finden zu können:

1. Ueberwiegend zellreiche Muskelpartieen, unwesentliche Bildung von Myogliafibrillen.

2. Uebergänge zu jugendlichen Muskelzellformen, Spindelzellen, insbesondere spindelige Kerne.

3. Degenerative Zellformen, sogenannte Sarkomzellen und deren Uebergangsformen zu Muskelzellen.

4. Mindestens unscharfe Grenzen zur Umgebung mikroskopisch, vielleicht sogar stellenweisen infiltratives Wachstum.

Bezüglich der Nomenklatur möchte ich ferner auf die Darlegungen Borst's in Aschoff's Lehrbuch (I. Teil) hinweisen. Borst nennt dort die heterotypen Geschwülste der Bindegewebsreihe fibromyo-glioblastisches Sarkom oder Fibroma, Myoma, Glioma sarcomatodes. Für zulässsig, wenn auch weniger empfehlenswert hält er die älteren Namen Fibrosarkom, Myosarkom, Gliosarkom; allerdings sollte dann in der Schreibweise "Fibrosarkom" etc. einerseits und "Fibro-Sarkom" etc. andererseits ausgedrückt werden, ob es sich um eine glioblastische, also um eine aus unreifem Gliagewebe sich entwickelnde Geschwulst oder um eine Kombinationsgeschwulst eines Glioms (resp. glioblastischen Sarkoms) mit einem gewöhnlichen Sarkom handelt. Auf den Gegenstand der vorliegenden Untersuchungen angewandt wäre also zu unterscheiden zwischen myoblastischem Sarkom = Myoma sarcomatodes $=$ Myosarkom einerseits und Myo-Sarkom andererseits. Neben den homootypischen 
und heterotypischen Geschwülsten erkennt Borst schliesslich eine Reihe von Geschwülsten an, die "sich biologisch wie maligne Tumoren verhalten, histologisch aber typisch gebaut sind". Für diese hält er die Bezeichnungen maligne Adenome, maligne Yyome usw. für geeignet. Die allein aus Muskelzellen bestehenden Geschwülste würden nach dieser Nomenklatur also eingeteilt in Myome, maligne Myome und Myosarkome (myoblastisches Sarkom, Myoma sarcomatodes).

Von Wichtigkeit für die Betrachtung zellreicher Nyome und Myosarkome scheint mir ferner eine Beobachtung Mallory's über das Verhalten der Myogliafibrillen in Myomen zu sein. Ich schicke voraus, dass die Untersuchungen der normalen Histologie über die Art und die Herkunft der Interzellularsubstanz im glatten Muskelgewebe noch nicht abgeschlossen sind. Besonders schwebt noch die Frage, ob die zwischen den glatten Muskelfasern befindliche, sich wie Bindegewebsfibrillen verhaltende Substanz von den in verhältnismässig geringer Anzahl sichtbaren Bindegewebszellen abstammt oder ob sie vielleicht ein Produkt der Muskelzellen darstellt. Anerkannt wird der von Haidenhain und von Benda getroffene Unterschied zwischen feinen Binnenfibrillen der glatten Muskelzellen und gröberen Grenzfibrillen. Letztere sollen eine spezitische Stützsubstanz der glatten Muskulatur darstellen. Nach den Beobachtungen Ogata's am geschlechtsreifen normalen Uterus sind die "Muskelfasern so dicht von feinsten Bindegewebsfibrillen umsponnen, dass eine förmliche Verschlinguug zwischen den Myofibrillen einerseits und den Bindegewebsfibrillen andererseits zu Stande zu kommen scheint". Uebergänge zwischen Myofibrillen und Bindegewebsfasern waren allerdings nicht nachzuweisen, auch waren zwischen beiden Lnterschiede in ihrem Verlauf festzustellen.

In Myomen ist dem Verhalten der Myogliafibrillen von Robert Meyer und von Mallory Aufmerksamkeit geschenkt worden. Die Myogliafibrillen verlaufen nach Meyer in wechselnder Zahl dicht den Zellsträngen angeschmiegt, ohne sich mit den interzellulären Fibrogliafibrillen zu vermischen. Ihre Ausläufer vereinigen sich mit benachbarten Zellen oder deren Fibrillen.

Mallory's Beobachtung war nun die, dass er in einem Tumor, der der übrigen Beschreibung nach vielleicht als ein Myosarkom angesprochen werden darf, bei den mehr unregelmässigen Zellen feinere und weniger gut entwicke]te Myofibrillen sah. 
Um die aben referierten Ansichten Robert Meyer's über das muskelzellige Sarkom, sowie die Beobachtung Mallory's nachzuprüfen, untersuchte ich, wie ich schon oben hervorhob, ausser sicher gutartigen zellreichen Myomen besonders jene vier Geschwülste, die im Freiburger Pathologischen Institut als maligne Myome oder Myosarkome oder doch mindestens sehr sarkomverdächtige Geschwülste diagnostiziert worden waren. Gefärbt wurden die Präparate mit Hämatoxylin-Eosin, Alaun-Karmin, van Gieson, Methylgrün-Pyronin, Azur II-Eosin sowie nach der Mallory-Ogata'schen Methode und deren Modifikation durch Daëls. Zur Technik der letzteren Methoden ist anzugeben, dass Ogata in Schnitten durch normale Uterusmuskulatur der verschiedenen Altersstufen bei seiner Färbung, Kerne, Kernkörper, Myoglia und elastische Fasern rot, Muskclfasern gelblich, Bindegewèbe blau gefärbt sah. Daëls, der den Wert dieser Färbung an normaler Uterusmuskulatur von Föten und Erwachsenen und an Uterusgeschwülsten nachprüfte, gibt verschiedene Färbung der Myofibrillen an, die zum Teil auf seiner Anwendung verschieden stark abgestufter Zusammensetzung der Farblösung beruhen mag. In einigen Präparaten färbten sich die Myofibrillen gelb, auch gelb-rot, in anderen blau. Das Bindegewebe färbte sich auch in seinen Präparaten immer blau.

Ich komme nunmehr zu meinen Untersuchungen und beginne mit der Muskulatur eines normalen puerperalen Uterus.

Makroskopisch lässt ein Schnitt durch den Uterus ein Netz aus dicken bindegewebigen Strängen erkennen, die das eigentliche Muskelgewebe in schmale, kleine Felder zerlegen. Am dichtesten und am deutlichsten sichtbar liegen diese Stränge in der mittleren, zahlreiche breite Gefässe führenden Muskelschicht.

Gröbere Verschiedenheiten in Farbe oder Struktur sind an den verschiedenen Stellen der Muskulatur nicht wahrzunehmen.

Mikroskopisch sieht man ein Geflecht aus stärkeren Muskelbündeln und einzelnen Muskelzügen, die durch dickere oder feinere Bindegewebssepten getrennt werden.

Die Richtung der Züge ist meist zirkulär, in der subserösen und submukösen Muskelschicht verlaufen die Zïge überwiegend in der Längsrichtung des Uterus.

Die Muskelzellen liegen in den einzelnen Zügen durch Interzellularsubstanz getrennt regelmässig nebeneinander. Sie haben die Gestalt einer langen Spindel und färben sich nach Hämatoxylin-Eosin violett.

Die sich blau färbenden Kerne sind alle gleichartig. Sie haben die Form eines langen Stäbchens mit verhältnismässig scharfen Ecken an den Enden. Sie sind alle schmal und lang, das Verhältnis von Breite zu Länge beträgt etwa 1:20. Irgend erhebliche Abweichungen in der Gestalt kommen nicht vor. Kernteilungsfiguren habe ich nirgends 
in der Muskulatur finden können. Regressive Veränderungen der Muskulatur fehlen.

Das Verhalten der Fibrillen wurde im Mallory-Ogata-Präparat (Daels'sche Modifikation 1:2) studiert. In diesem Präparat erscheinen Kerne und Kernkörperehen, die als feine Pünktchen wahrzunehmen sind, rot.

Der Zellleib ist blassblau bis gelblich gefärbt und lässt an den längsgetroffenen Stellen eine feine Streifung in der Längsrichtung erkennen. Zwischen den Zelleibern liegen blau gefärbte dünne Fibrillen, die zum Teil den für Bindegewebe charakteristischen welligen Verlauf zeigen, zum Teil aber auch mehr gestreckt verlaufen.

Diese Fibrillen der Interzellularsubstanz sind durchweg von der gleichen Dicke und Länge. In den quergetroffenen Partien bilden die blauen Fibrillen ein zierliches Netzwerk, in dem nur sehr wenige ihm angehörige Zellen liegen. In den Maschen des Netzwerks, aber ohne mit diesem in direkte Verbindung zu treten, liegen die quergetroffenen Muskelfasern.

Meine nach Mallory-Ogata gefärbten Schnitte normaler Uterusmuskulatur bestätigen nach dem Gesagten insofern völlig die Befunde Ogata's, als auch ich in der Uterusmuskulatur eine feine Umspinnung jeder einzelnen Muskelfaser durch feinste Fibrillen feststellen konnte.

Es folgen die untersuchten zehn unverdächtigen Myome. Von diesen zehn waren neun durch Operation gewonnen und hatten wenigstens klinisch kleine Metastasen gesetzt, bei dem zehnten bei einer Sektion gefundenen, konnten Metastasen mit absoluter Sicherheit ausgeschlossen werden.

Es handelte sich teils um solitär, teils um multipel aufgetretene Myome, die in den verschiedenen Teilen der Uteruswand ihren Sitz hatten. Thre Grösse variierte zwischen Haselnussgrösse bis über Mannskopfgrösse. In allen Fällen war die Grenze gegen das umgebende Uterusgewebe überall scharf. Einige der Geschwülste zeigten schon makroskopisch regressive Metamorphosen.

Makroskopisch liessen alle Myome einen mehr oder minder deutlichen Aufbau aus einzelnen Wirbeln erkennen. Am deutlichsten sieht man diesen charakteristischen Aufbau bei den bindegewebsreichen harten Myomen, während die bindegewebsarmen, weichen auf ihrer Schnittfläche mehr ein gleichmässiges Bild von weiss-gelblicher Farbe bieten. Im allgemeinen zeigen die Myome mit grossem Zellgehalt nur wenig Interzellularsubstanz, während bei den zellarmen das Bindegewebe stärker entwickelt ist. Uebrigens wechseln auch im einzelnen Myom zellreichere und zellärmere Partien, in denen das Bindegewebe dem Zellgehalt wie erwähnt entsprechend vorhanden ist.

In allen Myomen liegen die Zellen in Zügen angeordnet, die sich mehr oder minder regelmässig durchflechten. Den regelmässigsten Aufbau zeigen auch mikroskopisch die harten Myome.

Die einzeInen Muskelzellen resp. ihre Kerne bieten in den verschiedenen Myomen ein innerhalb gewisser Grenzen variierendes Aussehen dar. Die Zellleiber haben die Gestalt einer langen schmalen 
Spindel und färben sich mit Hämatoxylin-Eosin violett, nach van Gieson gelblich. Die Kernform lässt sich am besten an den Stellen erkennen, wo Muskelbündel genau längs getroffen sind, also keine Täuschungen durch Schräg- oder Querschnitte entstehen können. Die Grösse der Kerne übertrifft sowohl in der Länge als besonders auch in der Breite die der normalen Uterusmuskulatur. Die Form ist im allgemeinen die eines mehr oder minder langen Stäbchens. Bei der Betrachtung mit Immersion zeigt sich jedoch, dass die Kerne durchaus nicht in allen Myomen die regelmässige Form mit scharfen Ecken haben. Vielfach sind die Ecken abgerundet und der Kern in der Mitte meist breiter, so dass oft eine Mandelform entsteht. Ebenso zeigen die Kerne der verschiedenen Myome Differenzen in der Grösse. Im allgemeinen könnte man in den Myomen von zwei Typen von Kernformen sprechen. Die einen, die vorwiegend in den harten bindegewebsreichen Geschwülsten zu sehen sind, sind alle gleichartig. Sie haben ziemlich regelmässige Form, sind sehr lang und schmal. Die anderen, vorwiegend in den bindegewebsarmen Geschwülsten auftretend, sind im allgemeinen kürzer und breiter. Sie erscheinen heller als die vorigen und variieren auch mehr in der Gestalt, indem breitere kürzere Kerne mit etwas weniger breiteren wechseln. Auch kommen geringe Abweichungen von der regelmässigen Form vor. Die Geschwiullste oder Geschwulstpartien, die diesen zweiten Typ der Zellformen zeigen, lassen einen weniger straffen Aufbau der Zellzüge erkennen. Die Zellen liegen nicht so genau ausgerichtet parallel zu einander, sondern gruppieren sich loser und gewissermassen zwangloser.

Vereinzelte Kernteilungsfiguren liessen sich bei 3 Geschwülsten nachweisen, bei der einen von diesen war sogar in jedem 10.-- 15. Gesichtsfeld bei Immersion eine zu finden. Die letztere Geschwulst, von der Figur 1 einen Schnitt darstellt, war ein grosses hartes Myom ohne regressive Veränderungen. Die Zellen besassen lange schmale Kerne und waren zu einzelnen ziemlich gleichmässig sich durchflechtenden Zügen angeordnet.

In 5 Fällen wurden teils vereinzelt teils sehr zahlreich Zellen mit Kernen gefunden, die sich in Karyorrhexis befanden. Diese Bilder sehen oft den Kernteilungsfiguren ähnlich und können bei oberflächlicher Betrachtnng mit diesen verwechselt werden. Sie unterseheiden sich jedoch von den Kernteilungsfiguren durch die grössere Form und ungleiche Grösse der einzelnen Teilchen, sowie durch die in einzelnen Abschnürungs kügelchen noch erkennbare Chromatinstruktur. Diese in Karyorrhexis befindlichen Zellen gehören wohl zu den Lymphozyten. Wenigstens macht es ihre den übrigen Lymphozyten in den Präparaten entsprechende Lage wahrscheinlich. Auch zeigt bei manchen Lymphozyten der Kern einen etwas unregelmässigen Rand und könnte als beginnende Karyorrhexis aufgefasst werden.

Ausser den erwähnten fast in allen Präparaten nachweisbaren Lymphozyten wurden in sechs Fällen mehr oder weniger zahlreiche Mastzellen gefunden.

Bei drei Myomen wurden endlich Plasmazellen festgestellt, die vereinzelt zwischen den Muskelzellen lagen.

Von regressiven Veränderungen war mikroskopisch bei sieben beginnende oder auch schon weiter fortgeschrittene hyaline Degeneration zu schen. Bei zweien wurde eine ödematöse Auflockerung mancher Gewebspartien beobachtet. 
Die hyaline Degeneration des Geschwulstgewebes war vielfach von einer gleichzeitigen hyalinen Degeneration der Wand der kleinen Lymphgefässe begleitet. Sie trat öfters gerade in den an diese Lymphspalten angrenzenden Partien auf.

Die Art der allmählich fortschreitenden hyalinen Degeneration liess sich an folgenden Bildern erkennen, Die Stellen, die das noch unveränderte Geschwulstgewebe repräsentieren, zeigen gleichmässig dichtgelagerte Kerne und wenig Interzellularsubstanz. Auf dem nächsten Bild sieht man die Kerne in Reihen zusammengedrängt, während gleichzeitig die Interzellularsubstanz $z$ wischen diesen Reihen breiter wird. Die Kerne unterscheiden sich zunächst noch nicht von denen des ur-

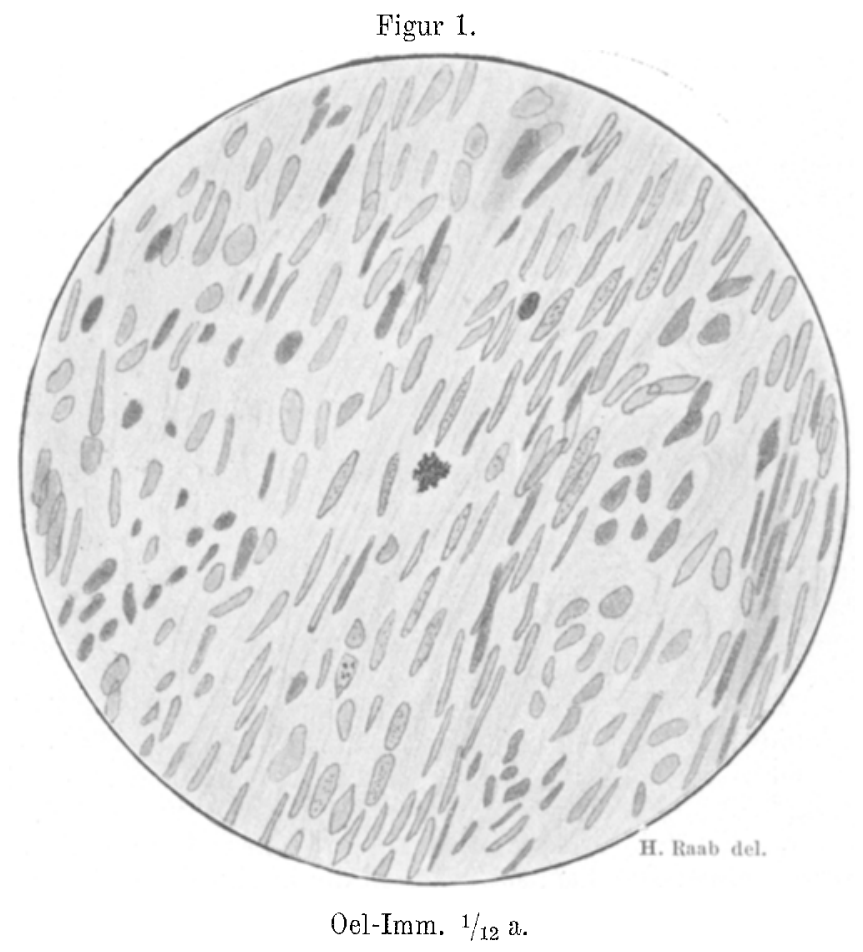

sprünglichen Geschwulstgewebes, damn aber, wo der Prozess schon weiter fortgeschritten ist, färben sie sich weniger distinkt, sehen wie verklumpt aus, und verblassen dann mehr und mehr, je näher sie den schon völlig hyalinen Teilen liegen.

Die Interzellularsubstanz ist indessen immer breiter geworden und schliesslich zu einer homogenen Masse konfluiert, die das Hyalin darstellt. Darin liegen noch einzelne Kerne und Kernreste, die noch ihre ursprüngliche Anordnung zu Reihen erkennen lassen, indem sie gewissermassen wie allmählich sich verjüngende Strahlen in die hyalinen Partien eindringen.

Das Hyalin selbst färbt sich nach Hämatoxylin-Eosin gleichmässig rosa, bei der Färbung nach van Gieson gleichmässig orange-rot.

Die hyalinen Veränderungen wurden vorwiegend bei den zahlreichen Myomen gefunden, während sie bei einem ziemlich binde- 
gewebsreichen Myom völlig fehlten. Auch schien es mir, dass innerhalb des einzelnen Myoms die hyaline Degeneration vorzugsweise in den sehr zahlreichen Partien auftrat, die gerade auch die grösseren und mehr polymorph gestalteten Kerne enthielten.

In einem Präparat wurde an einer Stelle, die beginnende hyaline Degeneration zeigte, eine einzelne mehrkernige Riesenzelle gefunden (Fig. 2). Die betreffende Geschwulst war ein grosses, weiches Myom, das mikroskopisch starken Zellreichtum aufwies. Die Kerne zeigten eine mässige Polymorphie im oben beschriebenen Sinne, indem zahlreiche Kerne grösser, breiter und heller waren und auch von der regelmässigen Gestalt abwichen. Die Figur gibt den Zellreichtum nicht

Figur 2.

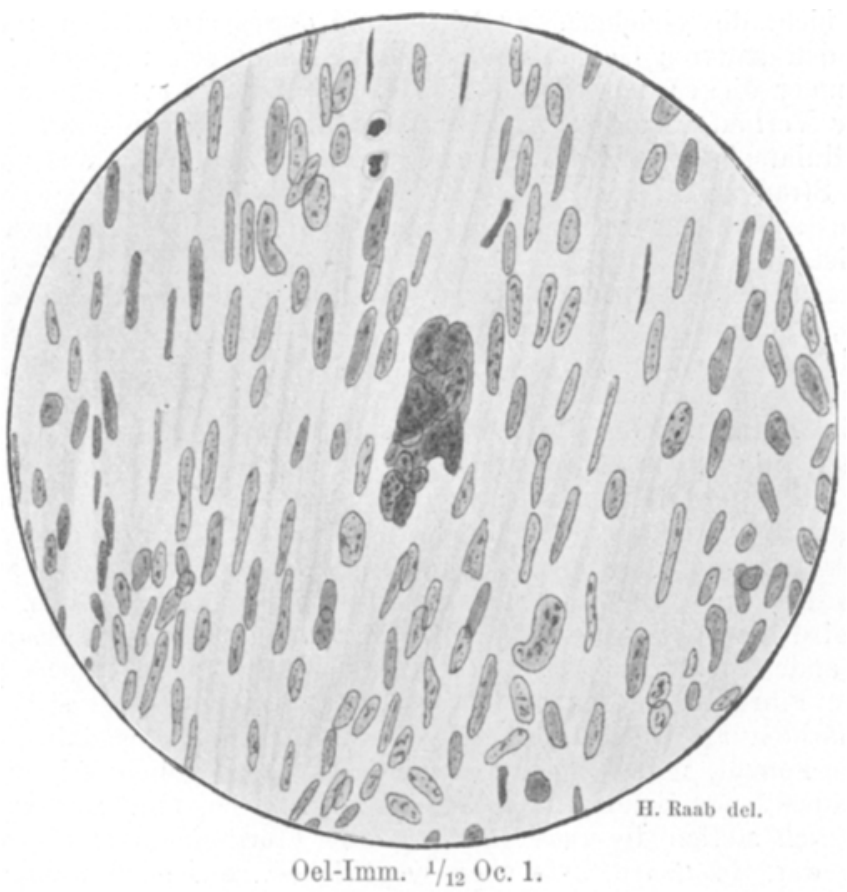

wieder und zeigt auch die Kernveränderungen nicht mehr so deutlich, wie sie an anderen Stellen zu sehen waren, weil der betreffende Sehnitt, wie gesagt, eine hyalin werdende Stelle der Geschwulst vor Augen führt. Immerhin lassen einige Kerne, mit denen von Fig. 1 verglichen, die Unterschiede erkennen.

Deutlich ist dagegen zu sehen, wie mit der beginnenden hyalinen Entartung die Interzellularsubstanz breiter wird und die Kerne in Reihen zusammenrücken.

Die Mallory-Ogata-Färbung ergab bei den Myomen folgende Resultate:

Die Kerne färbten sich deutlich rot. Die Fibrillen der sich schon blau färbenden Interzellularsubstanz verhielten sich in den verschiedenen Geschwülsten verschieden. 
In den Hyomen, die viel Interzellularsubstanz enthielten, bewirkten die Fibrillen oft eine diffuse Blaufärbung des Präparats. Die einzelnen Fibrillen waren oft schlecht zu erkennen, vielmehr sah das zwischen den Kernen liegende Gewebe verwaschen blau aus.

An den Stellen, an denen Fibrillen erkennbar waren, sah man zahlreiche dünne, sehr zierliche, lange Fibrillen, die gerade gestreckt parallel zur Längsrichtung der Zelle verliefen. ln anderen Myomen und zwar vorzugsweise in den zellreichen war eine viel deutlichere Scheidung zwischen Zellen einerseits und der Interzellularsubstanz andererseits zu erkennen.

In diesen Geschwülsen liegen nicht zahlreiche, feine Fibrillen zwischen den einzelnen Zellen, vielmehr werden Gruppen von Zellen durch wenige Fibrillen voneinander geschieden. Diese Fibrillen selbst zeigen nicht die gleichmässige Dicke und den gestreckten Verlauf, wie die in den anderen Geschwülsten, sondern sind von ungleicher Dicke, aber immer dicker und plumper als jene. Es fehlt vor allem der gestreckte Verlauf und die parallele Richtung der langen Fibrillen. Die Interzellularsubstanz bildet so ein grobmaschiges Netz aus plumpen, dicken Strängen, in dessen Maschen ein sehr zellreiches Gewebe liegt, in dem selbst nur wenig oder gar keine Interzellularsubstanz zu sehen ist.

Besondere Verhältnisse zeigen die Mallory-Ogata-Präparate in den Partien, die eine beginnende hyaline Entartung darstellen. Das eben beschriebene, noch erhaltene Gewebe ändert sein Aussehen in der Richtung nach den hyalinen Partien,

Die Kerne zeigen die Veränderungen in ihrer Lage und ihrem Aussehen, die schon bei den anderen Färbungen zu sehen waren. Sie rücken dicht in Reihen zusammen und färben sich weniger distinkt. Die oben beschriebene Verbreiterung der Interzellularsubstanz kommt dadurch zustande, dass die Fibrillen zusammensintern und so zwischen den fibrillenarmen Zellhaufen wenige breite Territorien bilden. Jedenfalls wird deutlich, dass diese verbreiterte Interzellularsubstanz aus nichts anderem besteht, als aus den schon ursprünglich vorhanden gewesenen Fibrillen, die nur noch mehr zusammengesintert sind. Diese blaugefärbten Fibrillenbündel rücken, je näher man den ganz hyalinen Partieen kommt, immer mehr zusammen, während gleichzeitig die Kerne verklumpen, um dann allmählich zu verblassen und $\mathrm{zu}$ schwinden. Schliesslich stellen die zusammengerückten Fibrillenbündel die hyalinen Massen dar, in denen, wie oben beschrieben, nur noch wenige Kerne und Kernreste liegen. Dieses Hyalin, das bei Hämatoxylin-, Eosin- und bei van Gieson-Färbung fast ganz gleichmässig gefärbte Massen darstellte, zeigt bei Mallory-Färbung noch eine feine fibrilläre Struktur, die auf seine Entstehung aus den Fibrillen der Interzellularsubstanz hinweist.

Nach der Beschreibung dieser unverdächtigen Myome komme ich zu den vier Fällen, die als Myosarkome oder als sarkomverdächtige Geschwülste diagnostiziert waren.

I.

Der erste Fall stellt einen Tumor einer Frau dar, der bei der ersten Untersuchung wie folgt beurteilt worden war: 
„Der ca. faustgrosse Uterus zeigt in der Isthmusgegend einen ins Lumen vorspringenden, kleinwalnussgrossen Tumor. Mikroskopisch erweist sich dieser als Myom mit sarkomatösen Partien. In diesen lassen sich deutliche Kernteilungsfiguren nachweisen, allerdings nicht sehr zahlreich, und Wucherungen von atypischen, spindeligen Zellen.

Die beiliegenden hasel- bis walnussgrossen Tumoren sind mässig zellreiche Myome“ (Prof. Schridde).

Makroskopischer Befund. Der aufbewahrte Uterus ist zerstückelt. Wie die einzelnen 6 Stücke zusammenpassen, ist nicht in allen Teilen mit Sicherheit festzustellen. Jedenfalls fehlen einzelne wenige Teile des Uterus. Das eine Stück, das anscheinend dem Fundus uteri entspricht, lässt deutlich Uteruswand erkennen, die eine mit Schleimhaut ausgekleidete Höhle umschliesst. Auf einer Seite besteht die Wandung aus einer flachen, etwa klein-hühnereigrossen Geschwulst, der auf ihrer der Vterus zugekehrten Seite zwei weitere kleinere Geschwülste, eine von Haselnussgrösse und eine von Mandelgrösse aufsitzen. Die hühnereigrosse Geschwulst ist ebenso wie die beiden kleineren auf der der Uterushöhle zugewandten Seite von Schleimhaut überzogen. Auf den Durchschnitten durch die Geschwülste erkennt man, dass das Geschwulstgewebe zu einzelnen Wirbeln angeordnet ist.

In dem anscheinend dem Fundus uteri entsprechenden Stück ragt in die Höhle ein haselnussgrosser Polyp mit breitem Stiel.

Ein anderes Stück des Präparats zeigt an einer Stelle, die anscheinend dem Isthmus uteri entspricht, auf der der Uterushöhle zugekehrten Seite eine runde, etwa haselnussgrosse Geschwulst, von der fast die Hälfte fehlt. Die Geschwulst ist makroskopisch gegen die Uteruswand scharf abgegrenzt. Auf dem Durchschnitt zeigt sie, ähnlich wie die anderen kleinen Geschwülste dieses Präparats, einen aus einzelnen Wirbeln bestehenden Aufbau.

Die übrigen aufbewahrten Stücke des Präparats sind Teile der Uteruswand, die keine Besonderheiten zeigen.

Mikroskopischer Befund. Der haselnussgrosse Tumor in der Isthmusgegend lässt bei schwacher Vergrösserung erkennen, dass er aus mehreren Knoten zusammengesetzt ist. Die Grenzen der einzelnen Knoten sind nicht überall scharf abgegrenzt, an manchen Stellen sieht man aber deutlich zwischen den Knoten Bindegewebe, das von breiten Lymphspalten durchsetzt ist. Auch Abgrenzung der Geschwulst gegen die Uteruswand wird meistens durch Lymphspalten gebildet, die sich auch in den anliegenden Schichten der Uteruswand zahlreich finden. Die Grenze ist im allgemeinen scharf; nur an einer Stelle ragt ein schmaler Streifen Geschwulstgewebe zwischen die nächstliegenden Spalten der Uteruswand hinein, von einem infiltrierenden Wachstum kann aber auch hier nicht die Rede sein.

Das Geschwulstgewebe ist bindegewebsarm. Es zeigt einen Aufbau ans deutlich erkennbaren, sich durchflechtenden Zügen. Die Züge selbst sind sehr zellreich, doch wechseln solche von stärkerer und geringerer ZelIdichte. Die Zellen liegen in den Bündeln dicht beieinander und sind an vielen Stellen genau ausgerichtet, dem Verlauf des Bündels entsprechend. Die Kernformen zeigen bei allen Geschwülsten dieses Präparats eine geringe Veränderung, die wohl auf die Fixierung zurückzuführen ist. Die Kerne sehen nämlich alle mehr oder weniger wie zerknittert aus; der Rand ist oft nicht glatt, sondern zeigt Knicke, die sich ins Innere des Kerns verfolgen lassen. Abgesehen von dieser 
artefiziellen Veränderung zeigen die Kerne keine Besonderheiten. Ihre Grösse übertrifft etwas die der ungebenden Uterusmuskulatur und variiert nur wenig. Ebenso ist ihre Form mit ganz geringen $\mathrm{Ab}$ weichungen überall die eines plumpen Stäbchens mit abgerundeten Ecken oder auch mehr zugespitzten Enden. In Sonderheit fehlt eine ausgesprochene Verbreiterung der Kerne.

Figur 3 stellt eine Partie aus diesem Geschwulstgewebe dar. Man erkennt die zu straffen Bündeln angeordneten, dichtliegenden Muskelzellen, deren Kerne alle die gleiche schmale, lange Form haben.

Die Geschwulst im Fundus uteri und die beiden ihr aufsitzenden, kleinen Geschwülste zeigen einen ähnlichen Aufbau, wie die vorige. Nur ist an manchen Stellen der Aufbau der sich dnrchflechtenden Zellzüge noch regelmässiger. Im übrigen wechseln auch hier sehr zellreiche mit zellärmeren Partien. Die Kerne zeigen ausser der beschriebenen artefiziellen Veränderung nur ganz geringe Abweichungen von

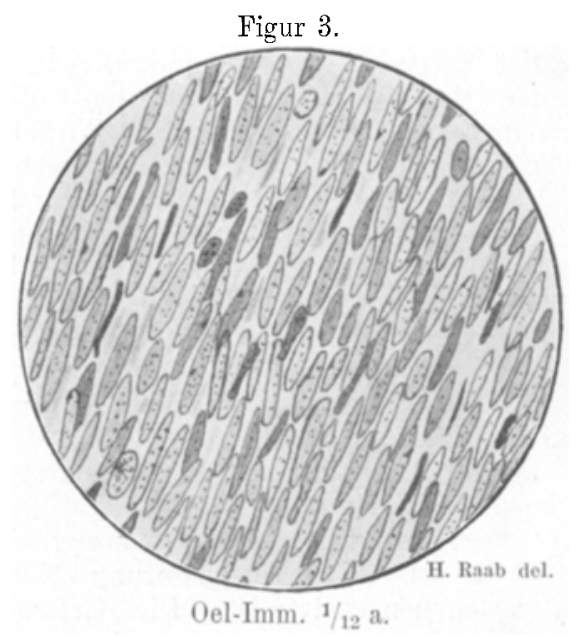

der gewöhnlichen Gestalt. Geringe Verbreiterung ihres Breitendurchmessers kommt in den zellreichen Partien vor. An einer Stelle der im Fundus uteri sitzenden Geschwulst erkennt man eine erst beginnende diffuse hyaline Entartung des interzellulären Gewebes.

Der makroskopisch als Polyp imponierende, in die Uterushöhle vorspringende Teil erweist sich mikroskopisch als Myom. Die Muskelzellen liegen auch hier in deutlich abgegrenzten Bündeln, die sich regelmässig durchflechten. Zellreichtum und Entwicklung des Bündelgewebes entsprechen den Verhältnissen in einem gewöhnlichen Myom. Das interzelluläre Gewebe zeigt eine beginnende, gleichmässig diftuse hyaline Entartung.

Ausser der an den zwei Stellen beschriebenen hyalinen Entartung sind nirgends im. Präparat regressive Metamorphosen zu finden.

In allen Geschwülsten dieses Präparats sind überall zerstreut sehr zahlreiche Mastzellen eingelagert, oft drei bis vier in einem Gesichtsfeld bei Immersion. Sie zeigen alle einen grossen Kern, die basophile Körnelung ihres Protoplasmas tritt am besten bei Methylgriun-Pyronin und bei Azur II-Eosinfärbung zutage. Eosinophil gekörnte Leuko- 
zyten wurden nur zweimal gefunden. Ferner liegen in allen Geschwülsten zerstreut Lymphozyten. Gelegentlich wurden Kerne gefunden, die in Karyorrhexis waren. Diese glichen durchaus den oben bei den gewöhnlichen Myomen beschriebenen. Auch sie sahen Kernteilungsfiguren ähnlich, konnten aber sicher von diesen unterschieden werden. Ihre Lage und das Verhalten anderer Lymphozyten machten es gleichfalls in diesem Präparat sehr wahrscheinlich, dass sie zu den Lymphozyten gehören. Eine sichere Kernteilungsfigur (Mitose) wurde in zahlreichen darauf durchsuchten Präparaten nur einmal, und zwar in der Geschwulst im Fundus uteri gefunden.

In zwei Schnitten, die zelldichtere Partien getroffen hatten, waren einige wenige mehrkernige Riesenzellen nachzuweisen. Diese Riesenzellen unterschieden sich durch Gestalt und tinktorielles Verhalten von der oben beschriebenen mehrkernigen Riesenzelle in einem gewöhnlichen gutartigen Myom. Die Kerne waren etwas kleiner als jene, färbten sich blasser und weniger distinkt. Sie lagen ferner nicht auch nebeneinander, sondern waren in einer Anzahl von etwa vier bis sieben zu einer Reihe angeordnet. Der ebenfalls blassgefärbte Zellleib zeigte noch die spindelförmige, wenn auch vergrösserte Gestalt. Dem ganzen Aussehen nach schien es sich im Vergleich zu der beschriebenen Riesenzelle in dem gutartigen Myom um eine jugendlichere Form zu handeln.

Plasmazellen waren in diesem Präparat nirgends zu finden.

Bei Mallory-Ogata-Färbung zeigen die Myome dieses Präparats relativ reichliche Fibrillenbildung. Zahlreiche feine Fibrillen, zum Teil auch etwas dickere, bilden ein feinmaschiges Netz, sodass jede einzelne Zelle umschlossen wird. An manchen Stellen entsteht dadurch eine diffuse Blaufärbung des Gewebes, an den anderen, wo die einzelnen Fibrillen zu erkennen sind, zeigen diese einen gestreckten Verlauf.

Nach der vorliegenden Beschreibung handelt es sich also um eine Geschwulst aus Muskelzellen, die in den einzelnen Knoten verschiedenen Gehalt an Muskelzellen und an Bindegewebe aufweist. Bei den zellreichsten Partien kann man von sehr schwacher Entwicklung des Bindegewebes sprechen. Die Struktur des Gewebes ahmt die normaler glatter Muskulatur nach. Die Kernveränderungen der sogenannten Sarkomzelle fehlen ganz oder sind nur gerade angedeutet. Kernteilungsfiguren sind sicher nur ganz selten im Präparat. Mehrkernige Riesenzellen konnten nur vereinzelt gefunden werden. Hyaline Veränderungen fehlen oder sind in ganz geringem Grade aufgetreten.

Was die Abgrenzung gegen die normale Uterusmuskulatur anbelangt, so könnte man vielleicht schon an ein eben beginnendes infiltratives Wachstum denken. Der diesbezügliche Befund ist aber so gering, dass er meines Erachtens noch innerbalb der normalen Wachstumsverhältnisse von Myomen liegt.

Nach diesem Befund ist die Geschwulst als ein zellreiches Myom anzusprechen. Unterstützt wird die Diagnose durch den 
weiteren klinischen Verlauf. Nachforsehungen haben ergeben, dass die vor 9 Monaten operierte Trägerin der Geschwulst noch lebt und bis heute rezidivfrei ist.

II.

Fall 2 ist ein Tumor, der bei der ersten Untersuchung im Freiburger Pathologischen Institut wio folgt beurteilt war:

„Es handelt sich um ein zellreiches, aber noch deutlich die Muskelfaserstruktur überall widerspiegelndes Myom. Dasselbe kann wohl als schnell wachsendes, eventuell malignes Myom, aber noch nicht als Myosarkom bezeichnet werden. Dazu fehlt die Atypie der Geschwulstzellen." (Geheimrat Aschoff).

Klinisch war die Geschwulst ohne grosse Blutungen innerhalb sehr kurzer Zeit rapid gewachsen. Dabei hatte starke Körpergewichtsabnahme stattgefunden, so dass Verdacht auf maligne Neubildung bestand.

Makroskopischer Befund: Es handelt sich um eine Geschwulst von kugelförmiger Gestalt und einem Durchmesser von $6 \mathrm{~cm}$. Sie sitzt in der rechten vorderen und seitlichen Wand des Uterus. Die ursprüngliche Form des Uterus ist durch die Geschwulst in der Weise stark verändert worden, dass die rechte Tubenmündung weit nach oben und links gedrängt wurde. Im gleichen Sinne wurde das Cavum uteri nach links verlagert. Die hintere Wand des Uterus ist sehr flach, während die vordere durch das Myom vorgewölbt wird.

Die Geschwulst selbst ist gegen die ungebende Uterusmuskulatur scharf abgegrenzt. Sie unterscheidet sich deutlich durch derbere Konsistenz, durch weissliche Farbe und sehnigen Glanz.

Auf ihrem Durchschnitt setzt sich die Geschwulst zusammen aus Wirbeln und richtigen Knoten, in denen man wieder eine Anordnung von weisslichen und graulichen Wirbeln wahrnimmt. Die Faserung verläuft in durchaus regelmässigen Zügen. Nirgend finden sich Stellen, die durch geringen sehnigen Glanz und Fehlen der geschilderten Struktur oder durch grössere Weichheit makroskopisch als Sarkom imponieren könnten.

Nach dem ganzen Verhalten der Geschwulst, nach ihrem Aussehen, ihrer Konsistenz und ihrer scharfen Abgrenzung gegen das normale Gewebe handelt es sich makroskopisch um ein gewöhnliches Fibromyom.

In der rechten vorderen Wand des Uterus sitzt in einer Entfernung von $5 \mathrm{~mm}$ von der grossen Geschwulst eine kleinere, die auf dem Durchschnitt einen Durchmesser von 8:10 mm hat. Sie zeigt makroskopisch dieselbe Struktur wie die grosse und scheint gegen die umgebende Uterusmuskulatur gleichfalls scharf abgesetzt.

Mikroskopischer Befund: Die Geschwulst besteht mikroskopisch aus einem im grossen und ganzen überall gleichartigen Gewebe. Nur in den zentralen Partien finden sich einige kleine Stellen, die regressive Metamorphosen aufweisen, welche man makroskopisch nicht nachweisen konnte. Hier geht das Tumorgewebe zum Teil in homogene hyaline Massen über, die sich bei Hämatoxylin-Eosin-Färbung rosa, bei Färbung nach van Gieson dunkelrot färben, zum Teil in ein myxomatöses Gewebe.

Das eigentliche noch nicht regressiv veränderte Tumorgewebe fällt schon bei schwacher Vergrösserung auf durch geringe Entwicklung des Bindegewebes und durch grossen Zellreichtum. Bei schwacher Vergrösserung lässt sich schon die makroskopisch sichtbare Anordnung zu grossen, durch feine Bindegewebssepten getrennten Zügen erkennen. Bei 
etwas stärkerer Vergrösserung spiegelt das Gewebe überall deutlich die Anordnung der Muskelfaserstruktur wieder, indem längsgetroffene Bündel mit schräg- und quergetroffenen wechseln. Dieses Gewebe scheint überall gleichartig entwickelt und wird von mässig viel Gefässen durchzogen.

Bei starker Vergrösserung handelt es sich um dichtgelagerte, spindelförmige Zellen, deren Protoplasma bei Hämatoxylin-Eosin-Färbung einen violetten Farbenton annimmt, bei van Gieson dagegen sich deutlich gelblich färbt.

Die Kerne sind grösser als die der umgebenden normalen Uterusmuskulatur. Bei der Betrachtung mit Immersion unterscheiden sie sich von den Kernen, wie sie sich in den meisten gewöhnlichen Myomen finden. Sie stellen sehr ausgesprochene Vertreter der oben beschriebenen Typs der grossen breiten Kerne dar. Sie haben nicht das schmale, stäbchenförmige Aussehen mit mehr oder minder scharfen Ecken, sondern sind bedeutend breiter, Sie erscheinen heller, sehen mehr bläschenförmig,

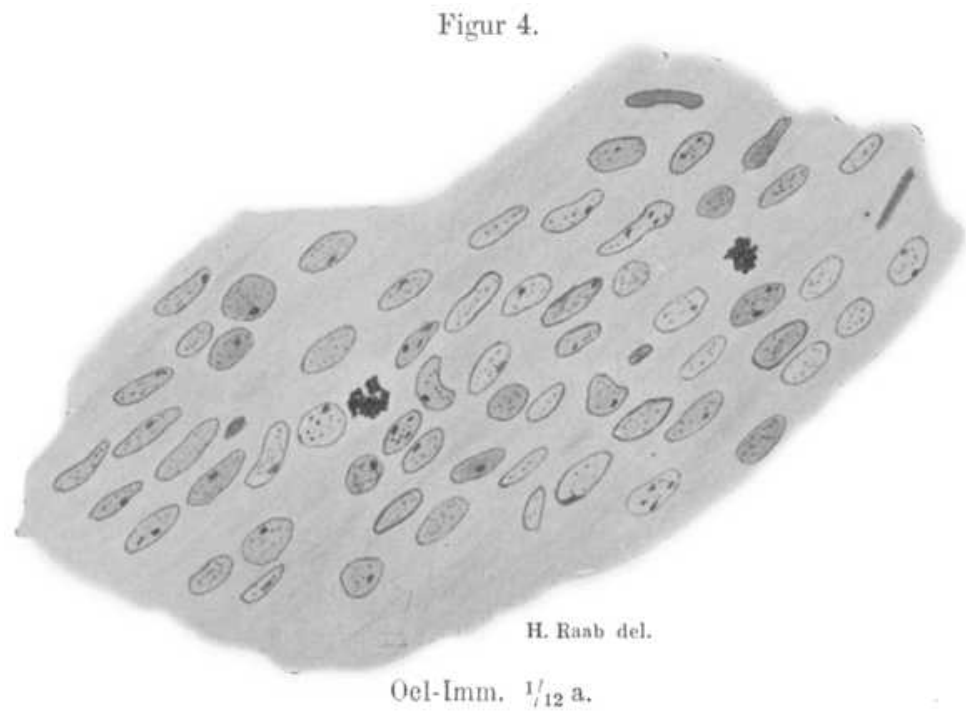

wie gequollen aus. Thre Gestalt ist rorwiegend mandelförmig. Die Kerne zeigen nur wenig merklichen Unterschied durch ihre wechselnde Grösse, durch ihre stärkere oder schwächere Färbefähigkeit, dann aber auch durch verschiedene Gestalt. Ausser der erwähnten Mandelform kommen Kerne vor von grosser ovaler oder fast runder Gestalt, manche zeigen eine oder mehrere Einkerbungen, einige haben Nierenform. Bei den Kernfärbungen (Hämatoxylin, Alaun-Karmin, Methylgrün-Pyronin) zeigen die Kerne ein zierliches Chromatinnetz. Es finden sich auch eine Menge feiner Netzknoten mit meist einem oder zwei Kernkörperchen, die sich basophil färben. Die Dicke der Kernmembran, die Anordnung der Chromatinfäden, die Grösse und Zahl der Kernkörperchen ist überall die gleiche. In den mehr nach der Peripherie gelegenen Teilen der Geschwulst lassen sich sehr wenige Kernteilungsfiguren finden. Meist muss man viele Gesichtsfelder durchsuchen, bis man auf eine Kernteilungsfigur trifft. In einem Gesichtsfeld waren zwei (Fig. 4). Es handelt sich um typische Mitosen, die alle gleiche Form aufweisen. 
Die Abbildung (Fig. 4) zeigt neben den Kernteilungsfiguren die charakteristischen Kernformen. In diesem Gesichtsfeld sind die Variationen in der Grösse, Gestalt und Färbefähigkeit deutlich erkennbar.

Riesenzellen oder Zellen mit mehreren Kernen sind in keinem Präparat zu finden. Nur an einer an der Peripherie der Geschwulst gelegenen Stelle wurde einmal eine Zelle mit einem sehr grossen Kern gesehen, dessen Aufbau in seinen Grundzügen aber absolut dem der anderen Zellen glich.

Was die Grenze der Geschwulst gegen die umgebende Uterusmuskulatur anbelangt, so wird die malkroskopisch sichtbare scharfe Abgrenzung auch mikroskopisch betätigt. In scharfer Linie ist das Tumorgewebe gegen die ersten zur Muskulatur gehörenden Bindegewebssepten abgesetzt, nirgends ist ein infiltrierendes Wachstum zu erkennen.

Die der Geschwulst nächstliegende Schicht der Uterusmuskulatur erscheint durch Druck verändert, die Kerne der Muskelfasern sind abgeplattet.

Die äussersten Schichten der Geschwulst fallen durch ganz besondere Zelldichte auf. Während wie oben erwähnt in den mehr nach der Peripherie gelegenen Teilen Kernteilungsfiguren liegen, sind solche in diesen alleräussersten zelldichten Schichten nicht zu finden.

In den peripheren Teilen der Geschwulst sind fast gleichmässig zerstreut massenhaft Mastzellen eingelagert, die besonders bei Azur IIEosin-Färbung durch ihre dunkelblau gefärbten Granula auffallen, Bei Methylgrün-Pyroninfärbung sind die Granula gelbrot gefärbt. Mastzellen von gleichem Aussehen finden sich in der die Geschwulst umgebenden Uterusmuskulatur fast noch zahlreicher.

Die Mallory-Ogata-Färbung ergibt bei diesem Tumor ähnliche Bilder wie bei dem vorigen. Vielleicht sind die einzelnen Fibrillen noch zahlreicher und noch feiner. Sie bilden ein sehr engmaschiges Netz und zeigen in den längsgetroffenen Partien einen sehr langen, ausgesprochen gerade gestreckten Verlauf.

Die kleine Geschwulst erweist sich mikroskopisch als ein stark fibröses Myom. Das Parenchym des Myoms ist zu zahlreichen dicken Bündeln angeordnet, die wie ein Knäuel durcheinander geflochten erscheinen und durch starke bindegewebige Stränge von einander getrennt werden. Die einzelnen Zellzüge unterscheiden sich von denen der grossen Geschwulst durch weit geringere Zelldichte und regelmässigere Anordnung der Zellen innerhalb der Bündel. Die Kerne gleichen im allgemeinen denen der gewöhnlichen glatten Muskelzelle. Sie sind schlanker als die der grossen Geschwulst und ziemlich gleichmässig gestaltet. Nur gelegentlich kommen Kerne vor, die etwas breitere ovale Gestalt haben, doch erreichen sie lange nicht die Formen der Kerne der grossen Geschwulst.

Auch in dieser kleinen Geschwulst finden sich zerstreut eingelagerte Mastzellen meistens in den peripheren Bezirken. Noch reichlicher Mastzellen kommen in der umgebenden Muskulatur vor, hier sieht man oft 8-10 in einem Gesichtsfeld bei Immersion. Im Bindegewebe der Geschwulst zerstreut liegen ziemlich grosse Herde von Lymphozyten. Vereinzelt wurden auch eosinophile Zellen gefunden. Kernteilungsfiguren konnten in dieser Geschwulst nicht gefunden werden. 
In diesem Fall handelt es sich um einen klinisch durch schnelles Wachstum und Körpergewichtsabnahme verdächtigen Tumor. Histologisch entspricht dem schnellen Wachstum ein starker Zellreichtum und wenig entwickeltes Bindegewebe. Die grobe Struktur des Muskelgewebes ist in der Geschwulst erhalten. Kernteilungsfiguren sind in geringer Zahl an der Peripherie zu finden. Die schon von früheren Autoren als für maligne Myome charakteristisch beschriebenen, von Robert Meyer als sekundäre Degeneration gedeuteten Veränderungen sind, wenn auch nicht sehr stark ausgesprochen, so doch sicher vorhanden. Wir sehen mässige Unterschiede in der Färbefähigkeit und eine Polymorphie, die neben einzelnen atypischen Formen, besonders in einer Vergrösserung der Kerne im Breitendurchmesser, die oft bis zu einer fast runden Gestalt führt, besteht. Hyaline Degeneration kommt nur an einer kleinen Stelle vor. Riesenzellen sind nicht vorhanden. Ein infiltrierendes Wachstum hat nicht stattgefunden. Metastasenbildung konnte wenigstens klinisch nicht nachgewiesen werden und man darf dem ganzen übrigen Befund nach wohl annehmen, dass auch keine stattgefunden hat.

Auch der weitere klinische Verlauf spricht gegen eine Metatastasenbildung. Die Myomträgerin wurde ein halbes Jahr nach der Operation untersucht und war rezidivfrei. Bald darauf erlag sie ihrem seit langem bestehenden schweren Herzleiden.

Alles zusammenfassend möchte ich diese Geschwulst trotz des Zellreichtums, trotz der vorhandenen Kernteilungsfiguren, trotz der Kernveränderungen nicht für ein malignes Myom halten, sondern für ein gewöhnliches zellreiches Myom. Der Vergleich mit den vorher beschriebenen unverdächtigen zellreichen Myomen zeigt, dass der Zellreichtum, das Vorhandensein mässig vieler Kernteilungsfiguren und die Kernveränderungen allein nicht genügen um ejn malignes Myom oder ein muskelzelliges Sarkom zu diagnostizieren. Der übrige Befund der Geschwulst spricht aber durchaus für eine benigne Neubildung.

Diese Auffassung des Falles stimmt überein mit seiner ersten Beurteilung, nach der die Geschwulst als ein „schnellwachsendes, eventuell malignes Myom, aber noch nicht als Myosarkom" bezeichnet werden kann. Der nicht wegen des histologischen, sondern 
wegen des klinischen Verhaltens angedeutete Verdacht auf Malignität hat sich ja auch durch den weiteren klinischen Verlauf nicht bestätigt.

III.

Der dritte Fall stellt einen Tumor dar, dessen 36 jährige Trägerin zur Zeit der Exstirpation im fünften bis sechsten Monat schwanger war. Die damalige Diagnose des Freiburger pathologischen Instituts lautete:

„Die Geschwulst erweist sich mikroskopisch als ein Myom, welehes zum Teil auffallend zellreich ist, atypische Zell- und Kernformen und relativ viele Kernteilungsfiguren aufweist, sodass die Diagnose auf Myosarkom gestellt werden muss." (Geheimrat Aschoff).

Makroskopischer Befund: Der gravide Uterus zeigt deutliche Dreiteilung. Dem Corpus uteri sitzt hinten rechts nahe dem Isthmus mit breitem Stiel eine faustgrosse Geschwulst auf. Ihre Masse betragen $5: 71 / 2: 8 \mathrm{~cm}$. Die Geschwulst ist von Serosa uiberzogen. Ihre Rückseite ist flach und ziemlich glatt. Auf der Oberfläche der gewölbten. vorderen Seite ragen zahlreiche hirse- bis linsengrosse und haselnussgrosse flache Höcker und beetartige unregelmässig begrenzte Erhabenheiten hervor, die gleichfalls von Serosa überzogen sind. Die Oberfläche der Geschwulst ist auf der Rückseite von annähernd gleichmässig gelblicher Farbe, auf der Vorderseite wechseln unregelmässig begrenzte Bezirke von schmutzig gelblicher Farbe mit solchen, die zum Teil bräunlich, zum Teil bläulich verfärbt sind. Die kleineren Höcker sind fast alle von schmutzig-gelblicher Farbe.

Auf ihrem Querschnitt besteht die Geschwulst aus weisslichem Gewebe, in dem eine Anzahl von etwa linsengrossen und grösseren, zackig begrenzten Partien von gleicher Beschaffenheit und grau-gelblicher Farbe sind; bisweilen erscheinen diese Stellen im Zentrum auch mehr dunkelbraun.

Das weisslich sehnig glänzende Gewebe zeigt eine feine Faserung, die an einigen Stellen eine Anordnung zu Wirbeln erkennen lässt, an den meisten aber strahlenförmig in die bräunlichen Stellen übergeht. Bei sehr genauer Betrachtung lässt sich erkennen, dass das weissliche Gewebe aus zwei verschiedenen Teilen besteht, die in kleinen, unregelmässig begrenzten Bezirken einander ablösen. Das eine Gewebe ist eine Spur mehr gelblich gefärbt, das andere sieht glänzend weiss aus und ist strahlenförmig gefasert.

An der Peripherie dicht unter der Serosa lassen sich einzelne isolierte, stecknadelknopf- bis erbsengrosse runde Knötchen erkennen. Diese bilden zum Teil die beschriebenen Höcker.

In der hinteren Uteruswand, in der Nähe des Stiels der grossen Geschwulst, sitzen zwei weitere kleinere Geschwülste im Abstand von $8 \mathrm{~mm}$. Die grössere liegt $6 \mathrm{~mm}$ unter der Serosa und hat einen Durchmesser von $6: 4 \mathrm{~mm}$, die kleinere liegt $1 \mathrm{~mm}$ unter der Serosa und hat einen Durchmesser von $3: 2 \mathrm{~mm}$. Eine letzte kleine Geschwulst wird in der hinteren linken Wand des Korpus festgestellt. Sie hat einen Durchmesser von $4: 3 \mathrm{~mm}$ und liegt dicht unter der Serosa, sodass sie diese etwa $1 \mathrm{~mm}$ weit hervorwölbt. 
Diese drei kleineren Geschwülste heben sich durch ihre Farbe deutlich von der umgebenden Muskulatur ab und zeigen makroskopisch eine scharfe Abgrenzung. Auf dem Durchschnitt lassen sie eine wellige Struktur erkennen, die die Geschwülste makroskopisch als gewöhnliche Fibromyome erscheinen lässt.

Mikroskopischer Befund: Die makroskopisch glasig und graugelblich aussehenden, eingelagerten Stellen erweisen sich mikroskopisch als ödematöses Bindegewebe. Die makroskopisch nur sehr schwer sichtbare Verschiedenheit des weisslichen Gewebes wird im gefärbten Präparat leicht sichtbar. Die glänzend weiss aussehenden Partien zeigen sich als hyaline Massen, die mehr gelblich gefärbten Partien dagegen als eigentliches Tumorgewebe.

Dieses Tumorgewebe besteht aus einem bindegewebsarmen, zellreichen Muskelgewebe, das zu einzelnen Zügen, die sich durchflechten, angeordnet ist. Die Anordnung dieser Züge unterscheidet sich nicht wesentlich von der eines gewöhnlichen Myoms. In den einzelnen Bündeln liegen die Zellen dicht zusammen. Die Zellen haben die Form einer langen, schmalen Spindel; ihr Protoplasma färbt sich bei Hämatoxylin-Eosin violett, bei Färbung nach van Gieson gelblich. Die Kerne gleichen denen des vorher beschriebenen Falls (2). Auch sie fallen auf durch ihre Grösse, die die der Kerne eines gewöhnlichen Myoms überschreitet, durch ihre breite, mehr ovale Form und das helle, bläschenförmige Aussehen, Sie lassen ein feines Chromatinnetz und ein bis zwei Kernkörperchen, die sich bei Methylgrïn-Pyroninfärbung rot färben, erkennen. Die Grösse der Kerne wechselt nur um ein geringes. Auch die Form ist im allgemeinen die gleiche, wenn anch einzelne Kerne kürzer und breiter, andere länger und schmaler erscheinen. Manche Kerne zeigen auch Einkerbungen.

Dieses so beschaffene Gewebe ändert sein Aussehen, je näher es den hyalinen Partien liegt. Die Interzellularsubstanz wird breiter, die Zellen werden zu einzelnen Reihen zusammengedrängt, färben sich weniger distinkt, sehen wie verklumpt aus, bis sie an der Grenze der hyalinen Partien allmählich verblassen.

- Einzelne Reste von Kernen und Protoplasma lassen sich bis weit in die hyalinen Massen hinein verfolgen. Die hyalinen Partien, die sich bei Hämatoxylin-Eosinfärbung rosa, bei der Färbung nach van Gieson leuchtend rot tingieren, lassen noch eine feine, strahlenförmige Faserung erkennen.

Kernteilungsfiguren sind nur ganz vereinzelt und nach langem Suchen zu finden. Ein deutlicher Diaster wurde an einer Stelle im Zentrum der Geschwulst an einer Grenze von myomatösem und hyalinem Gewebe gefunden.

Fig. 5 zeigt diese Kernteilungsfigur. Man erkennt ausserdem in diesem Bild, wie die Zellen an dieser hyalin werdenden Stelle sich in Reihen gruppiert haben und zum Teil nur noch undeutlich sich färben. In dem dazwischen liegenden, bereits hyalinen Gewebe sind noch Reste von Zellleibern und einigen Kernen erhalten. Von den noch erhalten gebliebenen Kernen zeigen manche die beschriebene Polymorphie.

Mastzellen waren auch bei Methylgrün-Pyronin-Färbung an keiner Stelle zu finden.

Die Mallory-Ogata-Färbung zeigt, dass in diesem Präparat die Interzellularsubstanz sich anders verhält als in den beiden vorigen. Man sieht nicht eine grosse Menge feiner interzellulärer Fibrillen, 
sondern ein grobmaschiges Netz, dessen Maschen dichte kleine Haufen von Zellen mit kaum vorhandener Interzellularsubstanz umschliessen. Die Maschen bestehen aus kurzen, plumpen Fibrillen, die zum Teil zusammengerïckt sind, und so kurze dünnere oder dickere Stämmchen bilden. Sehr gut, und deutlicher als bei den oben beschriebenen Geschwülsten ist in diesem Präparat der Uebergang des Geschwulstgewebes in die grossen hyalinen Partien zu sehen. Die Verbreiterung der Interzellularsubstanz entsteht durch das Zusammensintern von Fibrillen, während gleichzeitig die Kerne verklumpen und allmählich schwinden. Die Fibrillenbündel rücken im weiteren Verlauf immer dichter zusammen, bis sie die hyalinen, fast kernlosen Massen bilden. Auch in diesen

Figur 5.

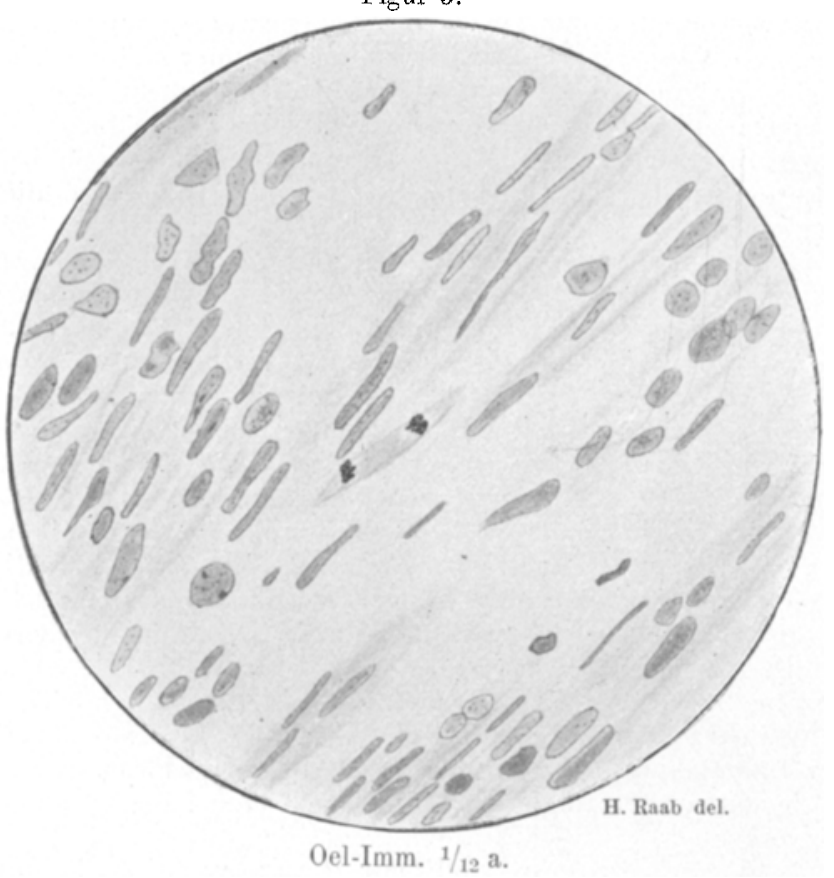

zeigt eine feine Streifung deutlich die Entstehung aus zusammengesinterten Fibrillen an.

In den ödematösen Partien der Geschwulst sind reichlich zerstreute Lymphozyten zu finden. Einzelne Muskelzellenbündel ragen auch in diese Partien hinein.

In einem der Peripherie der Geschwulst naheliegenden, isolierten Knoten erweist sich das makroskopisch wie die beschriebenen ödematösen Partien aussehende Zentrum mikroskopisch als ein zerklüfteter Hohlraum, dessen Ränder unscharf gegen die Umgebung abgesetzt sind. Der Hohlraum ist mit einer hellen, homogenen, bei Hämatoxylin-Eosin schwach rosa sich färbenden Masse ausgefüllt. In ihr finden sich einzelne runde Zellen mit rundem Kern, die reichlich eingelagerte, nach Sudan sich rot färbende Fetttröpfchen enthalten. Die Zellen des an den Hohlraum angrenzenden Myomgewebes enthalten gleichfalls zahl- 
reiche Fetttröpfchen. Bei Mlallory-Ogata-Färbung wird die den Hohlraum ausfüllende, sonst gleichmässig erscheinende Masse blau gefärbt und zeigt eine deutliche fibrilläre Zeichnung. Die darin liegenden verfetteten Zellen sind zum Teil in Reihen angeordnet.

Ein Schnitt von einer Stelle am Rande der Geschwulst lässt makroskopisch drei isolierte stecknadelkopf- bis erbsengrosse Knötchen erkennen, die von weisser Farbe und runder Gestalt sind. Mikroskopisch handelt es sich um kleine Muskelgeschwülste, die durch ein Bindegewebe, das breite Lymphgefässe führt, getrennt werden.

Die kleinen Myome gleichen in ihrem Aufbau der grossen Geschwulst und zeichnen sich besonders durch sehr gering entwickeltes Bindegewebe und verhältnissmässig grossen Zellreichtum aus. An manchen Stellen erscheint das Gewebe etwas aufgelockert, eigentliche regressive Veränderungen, besonders hyaline Entartung oder Verfettung, sind in ihnen nicht zu finden.

Die drei kleineren Geschwülste der hinteren Uteruswand zeigen typischen Myomaufbau. Das Bindegewebe ist stärker entwickelt als in der grossen Geschwulst, die Muskelbündel erscheinen regelmässiger durchflochten. Regressive Metamorphosen fehlen. Die Zellen liegen weniger dicht als die der grossen Geschwulst. Die Kernformen zeigen keine Atypien.

Die Grenze gegen die Uterusmuskulatur ist auch mikroskopisch absolut scharf.

Kurz zusammengefasst handelt es sich also in diesem Fall $1 I 1$ um eine Geschwulst, die ausgedehnte hyaline Veränderungen erfahren hat. Das erhalten gebliebene Gewebe stellt nach seinem morphologischen und tinktoriellen Verhalten zweifelloses Muskelgewebe dar. Es gleicht dem von Fall II in Zellgehalt und wenig entwickeltem Bindegewebe. Auch die Polymorphie der Kerne ist bis za einem gewissen Grade ausgesprochen. Kernteilungsfiguren sind nur in sehr geringer Zahl zu finden. Riesenzellen sind nicht vorhanden, auch nicht in den Grenzpartien zwischen erhaltenem Geschwulstgewebe und hyalinen Massen. Ein infiltrierendes Wachstum hat nicht stattgefunden, Metastasenbildung war klinisch nicht nachweisbar. Der weitere klinische Verlauf lässt Metastasenbildung ausschliessen. Die Frau ist jetzt, 8 Monate nach der Operation, völlig gesund. Die kürzlich vorgenommene gynäkologische Untersuchung konnte kein Rezidiv feststellen.

Der geschilderte Befund des von mir untersuchten Materials lässt aus den bei Fall II erwähnten Gründen die Diagnose Myosarkom nicht zu. Aus den von mir hergestellten zahlreichen Schnitten kann ich vielmehr für diese Geschwulst nur die Diagnose nicht malignes zellreiches Myom stellen. Da indessen seiner Zeit bei der ersten Untersuchung in dem zur Betrachtung ge- 
kommenen Schnitt Herr Geheimrat Aschoff neben Zellreichtum und atypischen Zell- und Kernformen "relativ viele Kernteilungsfiguren" - sodass die Diagnose Myosarkom gestellt werden musste - sah, will ich keine bestimmte Entscheidung treffen. Ich lasse es in der Schwebe, ob die Gesamtgeschwulst als zellreiches Myom, oder ob sie als Myosarkom aufgefasst werden muss.

IV.

Das Präparat von Fall IV war in der Sammlung als Myosarkom aufbewahrt worden.

Dieser letzte Fall ist von besonderer Bedeutung, insofern als die Trägerin der Geschwulst kurz nach der Operation zur Sektion kam und dabei eine Metastase in der Lunge festgestellt werden konnte. Die schon vorher aus der histologischen Untersuchung der exstirpierten Uterusgeschwulst gestellte Diagnose wurde dadurch bestätigt. Der Wortlaut dieser damaligen Beurteilung des Tumors lautete:

Mikroskopisch zeigt es sich, dass es sich um ein Myosarkom handelt. Die hauptsächlich aus Spindelzellen bestehenden Geschwulstwucherungen sind hauptsächlich um die Gefässe angeordnet und zeigen reichlich Riesenzellen. Weiter von den Gefässen entfernt findet sich entweder nekrotisches Geschwulstgewebe oder aber auch kernarmes Narbengewebe, das noch reichlich Geschwulstriesenzellen enthält. (Prof. Schridde).

Ich beginne mit der Beschreibung der Geschwulst.

Makroskopischer Befund: Es handelt sich um zwei, nach vorausgegangenem Morcellement durch supravaginale Amputation des Uterus gewonnene Korpustumoren. Klinisch war eine Geschwulst festgestellt worden, die von der Höhe der Interspinallinie bis links in die Symphysenhöhe und rechts bis in Nabelhöhe sich ansdehnte. Das in der Sammlung aufbewahrte Stück hat Kindskopfgrösse und ist von annähernd gleichmässiger kugeliger Gestalt. Die Durchmesser betragen $71 / 2-10 \mathrm{~cm}$. Diese Geschwulst wird in Scheiben von etwa $1 \mathrm{~cm}$ Dicke zerlegt. Auf den Duchschnitten präsentiert sich auf der einen Seite ein Teil der $2-21 / 2 \mathrm{~cm}$ dicken Uteruswand. Das übrige Gewebe gehört dem Tumor an und zeigt auf dem Durchschnitt ein buntes Bild, in dem Partien von verschiedenem Aussehen wechseln. Manche Stellen, die makroskopisch als das eigentliche Tumorgewebe imponieren, sind von gelblich-weisser Farbe und zeigen eine kaum sichtbare feine Faserung. Die Farbe dieser Partien ist nicht überall genau dieselbe. An den meisten Stellen ist sie mehr gelb; hier hat das Gewebe auch ein mehr homogenes Aussehen und unterscheidet sich vom übrigen Geschwulstgewebe auch in der Konsistenz, indem es weicher ist. An einzelnen kleinen Stellen ist das Gewebe mehr weisslich.

Dieses anscheinend eigentliche Tumorgewebe geht an vielen Stellen über in grössere Partien eines bräunlich verfärbten Gewebes, das kleinste Hohlräume bis Mohnkorngrösse enthält, die zum Teil mit einer bräun- 
lichen Masse gefüllt, zum Teil ungefüllt sind. Schliesslich sind im Präparat noch Stellen, die Stränge von weisslicher Farbe und sehnigem Glanz darstellen. Diese durchsetzen überall den Tumor und splittern an vielen Stellen sich zu einem feinen Netzwerk auf. Im Zentrum der Geschwulst ist die Anordnung dieser Stränge im grossen und ganzen radiär.

Die Grenze der Geschwulst gegen das ihr aufsitzende Stï̈ck der Uteruswand ist makroskopisch im allgemeinen scharf. Nur an manchen Stellen erscheinen einige der angrenzenden Bündel der Uterusmuskulatur abgesprengt und in die entstandene Lücke längliche etwa linsengrosse Stücke des Tumorgewebes vorgeschoben. Diese Teile des Tumorgewebes gehören zu den mehr weiss gefärbten; in ihrem Zentrum lassen sich aber vielfach Stellen erkennen, die den homogenen sehnig glänzenden entsprechen.

Mikroskopischer Befund: Bei Hämatoxylin-Eosin-Färbung erkennt man in dem Präparat wechselnde Bilder. Dabei zeigt sich, dass das eigentliche Tumorgewebe nur an sehr wenigen Stellen erhalten ist, überall sonst ist das Gewebe in regressiver Metamorphose.

Die makroskopisch sehnig glänzenden Partien, die meist strangförmig das Präparat durchsetzten, erweisen sich als hyalin. Das bräunlich verfärbte, kleinste Hohlräume enthaltende Gewebe besteht aus Tumorgewebe, das im Beginn der hyalinen Degeneration steht und das von zahlreichen Lymphspalten durchzogen wird. In diesen Partien erkennt man, dass die Wandungen der Lymphspalten hyalin sind. Die Kerne des hier vorhandenen Geschwulstgewebes sind in Reihen zusammengedrängt, die durch hyaline Bänder getrennt werden.

Die Teile des Geschwulstgewebes, die sich makroskopisch durch mehr gelbliche Farbe und reichere Konsistenz auszeichneten, stellen nekrotische oder nekrotisch werdende Teile des Geschwulstgewebes dar.

Die ursprüngliche Struktur des Tumorgewebes ist am reinsten erhalten in den makroskopisch mehr weisslichen Stellen an der Peripherie der Geschwulst und zwar in den in die Muskulatur hereinreichenden Zapfen. Dieses Gewebe ist sehr zellreich und zeigt geringe Entwicklung der Interzellularsubstanz. Die Muskelzellen sind teilweise zu kurzen Zügen angeordnet, an anderen Stellen lassen sich Züge nicht verfolgen, sondern man sieht kleinere Haufen von Zellen einfach nebeneinander liegen. Die Anordnung zu Zügen lässt sich hier deshalb nicht erkennen, weil von dem. Geschwulstgewebe überhaupt nur kleine Territorien erhalten sind, während die nächstliegenden Stellen schon regressiven Veränderungen verfielen. Das Protoplasma der Geschwulstzellen färbt sich nach Hämatoxylin-Eosin violett, nach van Gieson gelblich. Die Zellgrenzen sind schwer zu sehen, doch lässt sich an den Stellen, die regelmässigere Züge treffen, immerhin erkennen, dass es sich um Spindelzellen handelt. Die Kernformen ähneln sehr denen von Fall II. Sie haben meistens dasselbe bläschenförmige Aussehen und lassen ein Chromatinnetz mit feinen Knoten erkennen; deutliche Kernkörperchen heben sich dagegen in diesem Präparat nicht $a b$. Die Grösse der Kerne wechselt in geringem Masse. Die Kerne sind im grossen und ganzen von ovaler Form. Vielfach sind sie im Breitendurchmesser vergrössert und nähern sich so einer runden Form. In der Färbefähigkeit sind nur geringe Unterschiede zu beobachten; meist färben sich die grösseren breiteren Kerne etwas heller, die kleineren schmaleren etwas dunkler.

Am auffallendsten ist in diesem Präparat die grosse Anzahl von 
Kernteilungsfiguren. Es handelt sich dabei um typische Mitosen, von denen man durchschnittlich 2-3 in einem Gesichtsfeld bei Immersion findet.

Bei genauer Betrachtung sind ferner in diesen Partien einzelne grosse mehrkernige Zellen zu erkennen, die sich etwas blass tingieren und dadurch in dem zellreichen Gewebe nicht scharf hervortreten.

Fig. 6 zeigt das beschriebene junge, noch nicht regressiv veränderte Geschwulstgewebe aus einer Stelle an der Peripherie der Geschwulst. In einem Gesichtsfeld liegen 3 Kernteilungsfiguren. Die Kerne weisen deutliche Polymorphie anf. Ihre Lage zeigt die Anordnung der Zellen zu einem breiten längsgetroffenen Bündel.

Mehrkernige Riesenzellen sind in diesem Gesichtsfeld nicht zu sehen.

\section{Figur 6.}

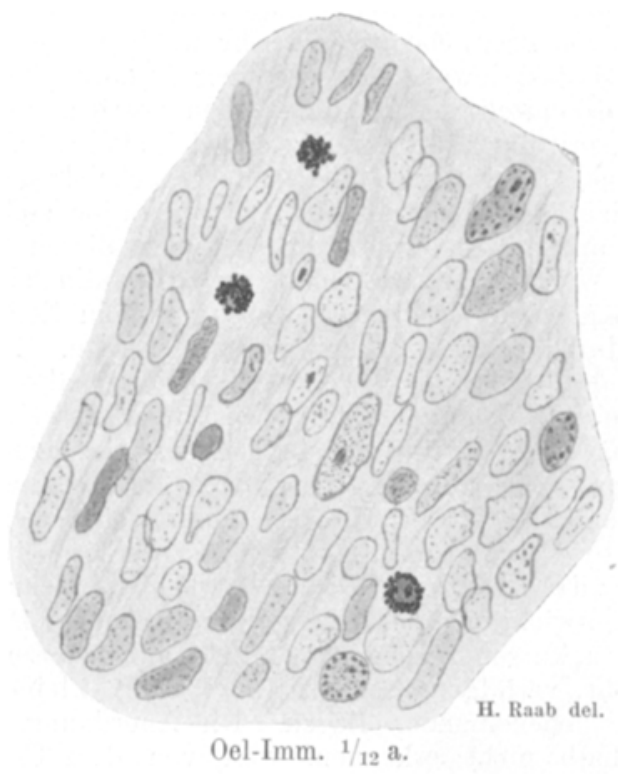

Dieses Geschwulstgewebe ändert sein Aussehen in den Partien, die an die hyalinen Stellen angrenzen. Hier fehlen die Kernteilungsfiguren, die Kerne färben sich dunkler, sind meist klein, von mehr runder Gestalt und sehen vielfach wie verklumpt aus.

Die Grenzpartien sind ferner besonders gekennzeichnet durch das Vorhandensein von ziemlich zahlreichen deutlich sichtbaren mehrkernigen Riesenzellen. Diese Riesenzellen sind von länglicher oder runder Gestalt und enthalten oft 6--15 Kerne, die sich dunkel färben. Gelegentlich werden Riesenzellen gefunden, in denen die Kerne in einer Reihe angeordnet liegen, so dass die ursprüngliche Spindelform der Zelle noch in der vergrösserten Zelle zu erkennen ist. (Diese Riesenzellen gleichen sehr den von Ulesco Stroganowa in ihrem malignen Uterusmyom Fig. 3 abgebildeten). In das hyaline Gewebe hinein lassen sich noch deutliche Ziige verfolgen, von denen Kerne oder Kernreste crhalten geblieben sind. An vielen Stellen im hyalinen Gewebe sind 
auch Riesenzellen sichtbar, die den beschriebenen gleichen, nur sind sie mehr oder weniger verblasst. Von den beschriebenen Randpartien weiter entfernt stellt dann das hyaline Gewebe in grösseren Bezirken eine homogene Masse dar, die sich bei Hämatoxylin-Eosin blassrot, bei van Gieson orange-rot färbt.

Fig. 7 zeigt eine Partie der Geschwulst, die schon ziemlich stark hyalin degeneriert ist. In dem bereits kernarmen hyalinen Gewebe liegen drei grosse, mehrkernige Riesenzellen, die der in einem gewöhnlichen unverdächtigen Myom beschriebenen Riesenzelle völlig gleichen.

Die übrigen noch erhaltenen Kerne des Geschwulstgewebes sehen wie verklumpt aus.

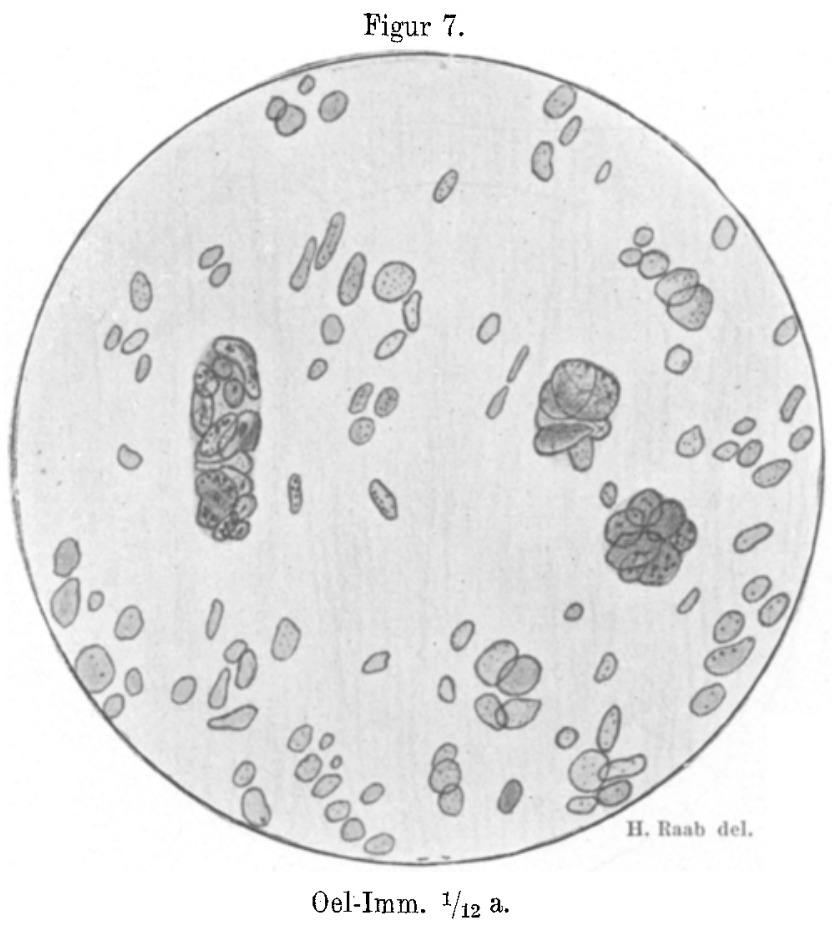

Die nekrotischen oder nekrotisch werdenden Partien der Geschwulst stellen ja nach dem Grad der Nekrose mehr oder minder homogene Massen dar, die sich nach Hämatoxylin-Eosin blassrötlich, nach van Gieson dunkelbraun färben. Manchmal offenbaren sich Reste der Struktur in einer gerade noch erkennbaren, feinen Streifung. An den meisten Stellen ist das Gewebe noch nicht völlig nekrotisch; dort sieht man massenhaft schmutzig und verwaschen gefärbte Kernreste und verklumpte Kerne, ebenso Reste von Riesenzellen. An anderen Stellen ist der Prozess weiter fortgeschritten und Kernreste sind nicht mehr erkennbar.

Sowohl in diesen nekrotischen und nekrotisch werdenden Partien, wie auch in den oben beschriebenen hyalin werdenden, finden sich zahIreiche kleine Gefässe, deren Wand hyalin ist und auch bei spezifischer Färbung keine elastischen Fasern zeigt. Das Lumen dieser Gefässe ist 
durch Thromben verschlossen, deren Fibrinnetz und Leukozyten noch gut erkennbar sind.

Plasmazellen oder Mastzellen sind nirgends in der Geschwulst zu finden.

Die Abgrenzung der Geschwulst gegen die angrenzende Uterusmuskulatur ist auch mikroskopisch mit Ausnahme der vereinzelten, schon makroskopisch sichtbaren, in das Uterusgewebe vorgeschobenen breiten Zapien überall scharf. Diese Zapfen bestehen in ihren Randpartien aus gut erhaltenem Geschwulstgewebe, in ihrem Zentrum ist das Gewebe schon hyalin degeneriert, Die Zapfen haben einige grosse Bïndel der Uteruswand abgesprengt und sind in einer Lymphspalte weitergewachsen. Die einzelnen Zapfen sind gegen die anliegende

Figur 8.

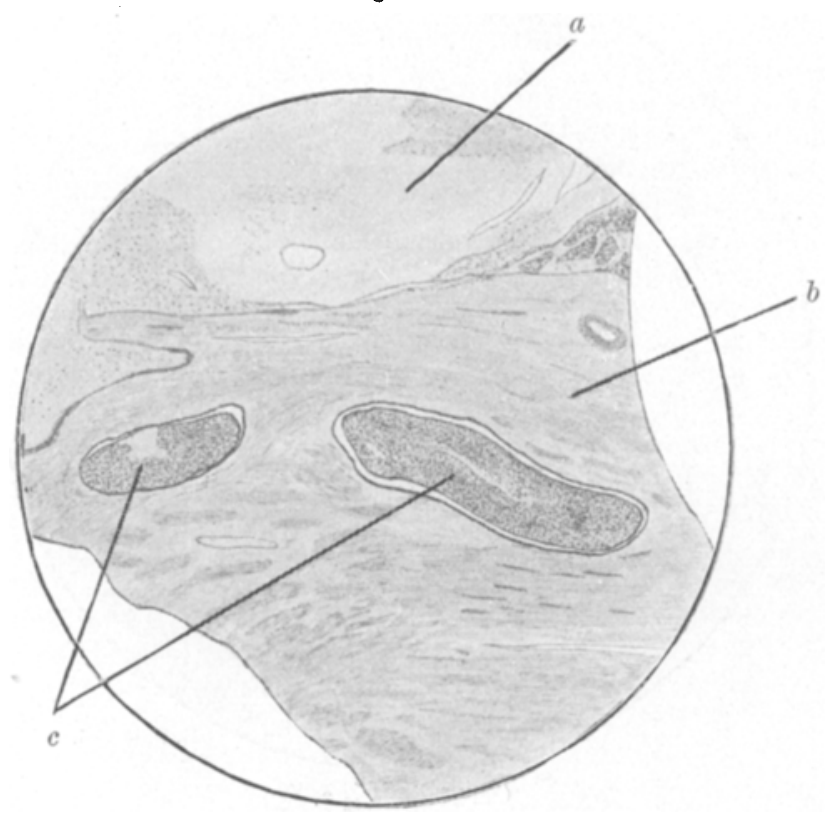

Muskulatur wieder scharf begrenzt, sodass von einem eigentlichen infiltrativem Wachstum nicht die Rede sein kann.

Diese Verhälnisse an der Peripherie der Geschwulst zeigt Fig. 8, die bei Lupenvergrösserung des Präparats gezeichnet wurde. Das obere Drittel (a) stellt Geschwulstgewebe dar, und zwar nekrotisches und nekrotisch werdendes, die beiden unteren Drittel $(b)$ die angrenzende Uterusmuskulatur. In dieser erkennt man in zwei Lymphspalten vorwachsend breite Zapfen von jungem Geschwulstgewebe (c). Die bereits einsetzende hyaline Degeneration dieses Gewebes ist an den darin befindlichen heller aussehenden Stellen erkennbar.

Die Mallory-Ogata-Färbung ergibt in diesem Präparat folgende Bilder. An den Stellen, die das noch unveründert erhalten gebliebene Geschwulstgewebe repräsentieren, sehen wir dieselbe Anordnung wie im 
Präparat III. Auch hier umspinnen nicht zahlreiche, feine Fibrillon in gleichartigem langgestreckten Verlauf jede einzelne Muskelzelle. Vielmehr werden Gruppen von Zellen, die unter sich durch wenige oder gar keine Fibrillen getrennt werden, geschieden durch Fibrillen oder Fibrillenbündel, die von ganz ungleicher Dicke sind. Diese Fibrillen sind aber immer dicker als die in den Präparaten I und II und verlaufen nicht so gestreckt und parallel zum Muskelzug wie jene. Sie sind auch viel kürzer and plumper und bilden ein Netz aus dicken Strängen, in dessen Maschen die fibrillenarmen Zellen liegen, oder ragen als kurze, sich schnell in weniger dicke Aeste aufsplitternde Stämmchen in die Territorien der Muskelzellen.

Beachtenswert sind die Stellen, die den Uebergang in die hyalinen Partien zeigen. Hier ändert das beschriebene Geschwulstgewebe sein Aussehen, indem die Muskelzellen nicht mehr gleichmässig verteilt liegen, sondern in Reihen zusammengedrängt werden, während gleichzeitig die interzelluläre Substanz allmählich breiter wird. Diese Verbreiterung geschieht, wie die Mallory-Ogata-Färbung zeigt, durch ein Zusammensintern der Fibrillen zu dicken Bündeln, die mit fortschreitender hyaliner Degeneration durch immer weitere Anlagerung von Fibrillen und Fibrillenbündeln breiter werden, Gleichzeitig verklumpen die Kerne des Geschwulstgewebes und schwinden mehr und mehr.

In dieser Zone der an Masse zunehmenden Interzellularsubstanz und der an Zahl abnehmenden Kerne liegen die mehrkernigen Riesenzellen. Anfänglich lassen die Riesenzellen noch deutlich die einzelnen Kerne erkennen, später verklumpen die Kerne zu sich dunkelfärbenden Gebilden, die keine Kernstruktur mehr zeigen. Schliesslich rücken die blaugefärbten Fibrillenbündel zu den dichten Massen zusammen, die bei gewöhnlichen Färbungen fast völlig homogen, hyalin erscheinen, die aber bei Mallory-Ogata eine deutliche fibrilläre Struktur zeigen.

Fig. 9 (Taf. XI) zeigt die beschriebenen Verhältnisse. In einem hyalinen Teil der Geschwulst hat sich das Geschwulstgewebe um ein Gefäss herum noch erhalten. Von hier aus ist der Uebergang des Geschwulstgewebes zu den hyalinen Massen zu sehen. Die nahe dem Gefäss noch unverändert liegenden Kerne werden weiter nach dem Hyalin zu in Reihen gedrängt, während die Fibrillen zu breiten Bändern zusammensintern. Dazwischen liegen mehrkernige Riesenzellen mit zum Teil schon pyknotischen Kernen. Nach den hyalinen Partien zu schwinden dann die Kerne der gewöhnlichen Zellen und der Riesenzellen, während die zusammengesinterten Fibrillenbündel die blaugefärbte hyaline Masse bilden, in der nur noch einzelne Kernreste erhalten geblieben sind. Wie auch aus der Zeichnung hervorgeht, kann man deutlich erkennen, wie die "hyalinen" Partien nicht aus homogenen Massen bestehen, sondern aus zusammengesinterten und verbackenen Fasern.

Diese Bilder sind wohl so zu deuten, dass da, wo die Myofibrillen zu breiten Bändern zusammengesintert erscheinen, es sich um ein Zeichen von Zelldegeneration handelt. Die Zellen gehen hier wohl wegen der schlechteren Ernährung zugrunde, während die Fibrillen erhalten bleiben. Die übrig bleibenden versuchen die Zellteilung, bringen es aber nur zur Kernteilung und werden so zu Riesenzellen. In solchen Riesenzellen sind anfangs die Kerne noch gut ausgebildet und gleichen denen der gewöhnlichen Zellen. Später, wenn die Degeneration in dem betreffenden Zellgebiet noch weiter fortschreitet, verklumpen die Kerne und werden pyknotisch. Wir haben dann die 
Riesenzellen mit tief dunkelgefärbten Kernen. Für die Berechtigung dieser Auffassung spricht, dass da, wo breite Bänder aus zusammengesinterten Fibrillen zu sehen sind, fast nur die Riesenzellen vorhanden und diese hier überhaupt am reichlichsten sind. Ferner dass dort, wo Riesenzellen mit pyknotischen Kernen liegen, fast keine anderen Zellen mehr zugegen sind, sondern nur noch blaue Territorien zusammengesinterter Fibrillen. Bei noch weitergehender Degeneration schwinden zuletzt auch die Riesenzellen, und man sieht nur noch die grossen unregelmässigen Bezirke, die man bei gewöhnlicher Färbung als hyalin zu bezeichnen pflegt.

Wenn man die Stellen aufsucht, die die ersten Anfänge der hyalinen Entartung der Interzellularsubstanz zeigen, so liegen diese schon in dem. fast noch unveränderten Geschwulstgewebe. Demnach handelt es sich nicht um eine nach Degeneration der Muskelfasern eintretende Verdickung des Bindegewebsgerüstes und nachträgliche hyaline Degeneration, sondern die hyaline Degeneration tritt gleichzeitig mit dem Schwinden der Muskelelemente oder sogar schon vorher ein und betrifft ausschliesslich die Fibrillen.

Die erwähnte Metastase der Geschwulst war ein erbsengrosses Knötchen in der Lunge. Histologisch glich es vollkommen der primären Geschwulst. Auch in ihm fanden sich zahlreiche Riesenzellen.

Es handelt sich also in diesem letzten Fall um einen aus Muskelzellen bestehenden Tumor. Dass die Geschwulstzellen tatsächlich Muskelzellen sind, wird durch ihr morphologisches und tinktorielles Verhalten sowie durch die wenigstens an manchen Stellen deutlich erkennbare Anordnung zu Zügen zweifellos bewiesen.

Die Geschwalst zeigt starken Zellreichtum und geringe Entwicklung des Bindegewebes und gleicht auch darin den vorher beschriebenen. Die Struktur des Gewebes ahmt die der normalen glatten Muskulatur nach, wenn auch dieses Verhalten wegen des nur in geringer Ausdehnung erhalten gebliebenen Geschwulstgewebes weniger deutlich sichtbar ist als in den anderen Geschwülsten.

Die Geschwulst hat sehr weitgehende regressive Veränderungen erfahren, insofern, als das Gewebe zum grössten Teil nekrotisch oder hyalin wurde. In dieser Beziehung hat sie Aehnlichkeit mit der in Fall III beschriebenen.

Die Genese der regressiven Veränderungen in der letzten Geschwulst hat man sich nach den beschriebenen Bildern wohl in folgender Weise vorzustellen. Sie beginnt mit der hyalinen Entartung, die wohl zuerst im perivaskulären Gewebe der kleinen Gefässe, dann aber auch in deren Nähe in der Interzellularsubstanz ihren Anfang nimmt. In einzelnen Abschnitten geht dieser Prozess ungestört weiter und führt zur vollständigen Hyalinisierung des 
Gewebes. In anderen Teilen der Geschwulst kommt es - vielleicht infolge der hyalinen Wandveränderungen - zur Entstehung von Thromben in den kleinen Gefässen. Nach Verschluss dieser kleinen Gefässe verfallen die von ihnen in ihrer Ernährang abhängigen Geschwultpartieen der Nekrose.

Die Kerne der Muskelzellen zeigen auch in diesem Falle die von R. Meyer für charakteristisch gehaltenen Veränderungen. Die Verbreiterung im Breitendurchmesser ist sicher vorhanden, ebenso die wechselnde Färbefähigkeit und eine gewisse Polymorphie. Die Kernformen gleichen denen von Fall II. Wenn überhaupt Unterschiede bestehen, so sind sie nur graduell, insofern, als hier die besprochenen Veränderungen vielleicht noch mehr ausgesprochen sind. Was die Kernteilungsfiguren anbelangt, so unterscheidet sich diese Geschwulst wesentlich von den drei vorigen. Bei jenen waren sie nur ganz vereinzelt und erst nach langem Suchen zu finden, bei dieser treten sie dagegen in den jungen Gewebspartieen gehäuft auf. In diesen Teilen der Geschwulst kann man durchschnittlich 2--3 in einem Gesichtsfeld bei Immersion sehen.

Wir haben hier also einen sehr schnell wachsenden Tumor vor uns, der ebenso schnell der regressiven Metamorphose verfällt und deshalb nur in den jüngsten Partieen an der Peripherie oder in der nächsten Umgebung von Gefässen die Morphologie des eigentlichen Geschwulstgewebes zeigt.

Von den 4 sarkomverdächtigen Geschwülsten kommt ferner diesem Fall allein zu das gehäufte Auftreten von mehrkernigen Riesenzellen, denn die bei Fall I erwähnten waren nur in ganz geringer Zahl vorhanden.

Dass Riesenzellen mit zahlreichen Kernen in Muskelgeschwülsten überhaupt auftreten können, zeigt die oben erwähnte, die in einem gewöhnlichen unverdächtigen Myom festgestellt wurde. Während dort aber nur eine einzige und bei Fall I nur einige wenige zu finden waren, treten hier die Riesenzellen in grosser Zahl auf. Zu beachten ist ferner, an welchen Stellen der Geschwulst die Riesenzellen angetroffen werden. In dem gewöhnlichen unverdächtigen Myom fanden sie sich in Gewebspartieen, die in Hyalinisierung begriffen sind. Bei Fall II lagen die sehr wenigen, anscheinend jugendliche Formen darstellenden mehrkernigen Riesenzellen an Stellen eines zelldichten Gewebes, das kein Hyalin aufwies. In der letzten Geschwulst sieht man mehrkernige Riesenzellen in zwei verschiedenen Geschwulstteilen lokalisiert. Einmal wieder in grosser 
Anzahl am Rand des hyalin degenerierten Gewebes, dann aber auch gerade in den peripher gelegenen jüngsten Partieen der Geschwulst. Fall IV hat also mit Fall I gemeinsam die Riesenzellen im nicht absterbenden Gewebe, unterscheidet sich aber von jenem dadurch, dass sie gerade am Rand der wachsenden Geschwulst und in grösserer Anzahl zu finden sind. Nach dem Geschilderten ist man vielleicht berechtigt, überhaupt zwei verschiedene Arten von Riesenzellen in den Geschwülsten anzunehmen, die sich in ihrer Genese unterscheiden. Einmal die im absterbenden Gewebe befindlichen Riesenzellen, die, wie oben ausgeführt, wohl dadurch entstehen, dass die absterbenden Zellen die Zellteilung versuchen, es aber nur bis zur Kernteilung bringen; die Riesenzellen wären also hier der Ausdruck einer Zelldegeneration. Von diesen Riesenzellen zu trennen sind die im jungen, wachsenden Geschwulstgewebe befindlichen. Die letzteren finden sich bei den von mir untersuchten Geschwülsten, abgesehen von den sehr wenigen, auch nicht gerade am Rande der Geschwulst liegenden Riesenzellen von Fall I, ausschliesslich in Fall IV.

Einen deutlichen Unterschied im Vergleich zu den vorher beschriebenen Fällen zeigt die Geschwulst ferner in ihrem Verhalten zur angrenzenden Uterusmuskulatur. Die Abgrenzung gegen die Uteruswand entspricht an den Stellen, wo Zapfen von Geschwulstgewebe in die Lymphspalten des Uterus vorwachsen, nicht mehr den normalen Verhältnissen. Bei längerem Bestehen der Geschwulst wäre wohl ein weiteres Vordringen in die Uterusmuskulatur, vielleicht auch ein destruktives Wachstum zu erwarten gewesen.

Endlich hat die Geschwulst eine Metastase, die histologisch den gleichen Aufbau zeigt, in die Lunge gesetzt.

Die nur dieser Geschwulst zukommenden Eigenschaften, das gehäufte Auftreten von Kernteilungsfiguren and mehrkernigen Riesenzellen, letztere auch in den jüngsten Geschwulstpartieen, das atypische Verhalten bei der Abgrenzung der Geschwulst und endlich die Metastasenbildung bestimmen mich, diesen Fall IV als eine maligne Muskelgesehwulst anzusprechen. Wenn man an der Unterscheidung zwischen malignen Myomen und Myosarkomen überhaupt festhalten will, möchte ich diese Geschwulst doch unbedingt zu den Myosarkomen rechnen. Denn es handelt sich hier nicht um eine mehr oder weniger zufällige Metastasenbildung eines histologisch gutartigen Tumors, 
sondern es liegen auch histologische Besonderheiten vor, die gewöhnlichen Myomen, auch den zellreichen, fehlen.

Nach dieser Untersuchung einer Anzahl gewöhnlicher, unverdächtiger Myome und der vier Geschwülste, die als maligne Myome bzw. Myosarkome, oder als dieser Malignität verdächtig diagnostiziert waren, möchte ich folgende Fragen aufwerfen.

Welche histologischen Merkmale sind entscheidend für die Diagnose eines Myosarkoms?

Können wir aus dem histologischen Bild eine sichere Diagnose auf die Bösartigkeit der Geschwulst stellen?

Ich halte dabei folgende Punkte für beachtenswert:

1. Zellreichtum und Struktur des Muskelgewebes.

2. Kernveränderungen.

3. Kernteilungen.

4. Verhalten der interzellulären Fibrillen (Hyalin).

5. Riesenzellen.

6. Abgrenzung der Geschwulst.

Was den Zellgehalt und die Struktur des Gewebes anbelangt, so fanden sich bei den untersuchten, unverdächtigen Myomen graduell verschiedene Gewebe. Dabei zeigten im grossen und ganzen die weniger zellreichen Myome eine stärkere Entwicklung des Bindegewebes, während in den zellreichen das Bindegewebe bzw. die Interzellularsubstanz mehr in den Hintergrund trot. Die Struktur des Muskelgewebes, das heisst, die Anordnung zu Zellzügen und weiter zu aus derartigen Zügen zusammengesetzten Wirbeln war bei den gewöhnlichen Myomen verschieden deutlich ausgesprochen. Die zellreichen Myome zeigen einen weniger regelmässigen Aufbau als die zellarmen.

Die früher als Myosarkome oder als verdächtig diagnostizierten Fälle I-III geben hinsichtlich des Zellgehalts folgenden Befund.

Alle drei sind sehr zellreiche Geschwülste, die nur geringe Entwicklung des Bindegewebes zeigen. Wesentliche Unterschiede im Vergleich zu den zellreichen Geschwülsten aus der Reihe der unverdäehtigen Myome sind in dieser Beziehung nicht zu finden. Ebenso verhält es sich mit der Struktur, die überall eine Gliederung in Wirbel und Züge erkennen lässt, wie sie die unverdächtigen, zellreichen Myome zeigen.

Der letzte Fall (IV) weist gleichfalls einen grossen Zellgehalt, 
verbunden mit spärlichem Vorbandensein der bindegewebigen Substanz auf. Die Struktur zeigt, soweit sie sich verfolgen lässt, keinen wesentlichen Unterschied im Vergleich mit den sarkomverdächtigen Geschwülsten und den zellreichen, unverdächtigen Myomen.

Nach diesem Befund können der von allen früheren Autoren bei der Schilderung maligner Myome und Myosarkome hervorgehobene starke Zellreichtum und die geringe Entwicklung des Bindegewebes, die nach Schlagenhaufer's Ansicht ein schrankenloses Wuchern des myomatösen Gewebes ermöglichen soll, nicht als Kriterien für die Diagnose Myosarkom gelten. Gewöhnliche, zellreiche Myome können das gleiche Verhalten zeigen.

Betrachten wir weiter bei den verschiedenen Geschwülsten die Kernveränderungen der "Sarkomzelle", denen R. Meyer bei der Schilderung des muskelzelligen Sarkoms so eingehende Beachtung schenkt. Die Untersuchung der gewöhnlichen, unverdächtigen Nyome zeigte, dass die einzelnen Geschwülste in dieser Beziehung sich verschieden verhielten. Bei Betrachtung mit Immersion haben die Kerne von Myomen durchaus nicht immer genau die Form eines langen Stäbchens mit scharfen Ecken, sondern variieren in jedem einzelnen Fall. Sehr oft sind die Stäbchen an den Enden abgerundet und nehmen dann eine mehr mandelförmige Gestalt an, besonders, wenn sie an den Enden abgerundet sind. Man könnte vielleicht von zwei Typen der Kernformen sprechen. Der eine zeigt die mehr regelmässige lange Stäbchengestalt, der andere wird durch die Abweichungen von dieser Gestalt im Sinne der beschriebenen Polymorphie dargestellt. Im übrigen bleibt die Grundform trotz der Abweichungen doch die des gewöhnlichen Kerns der glatten Muskelzelle. Man könnte die bei jedem Myom und auch innerhalb des einzelnen Myoms etwas anders aussehenden Kerne vergleichen mit verschiedenen Individuen ein und derselben Rasse, von denen auch nicht eins genau aussieht wie das andere, die aber doch alle einen gemeinsamen gleichen Typ aufweisen.

Von den drei sarkomverdächtigen Geschwülsten repräsentieren die Kerne des ersten Falls den Typ der regelmässigen Stäbchenform. Die beiden anderen zeigen die schon bei den gewöhnlichen Myomen beschriebene Polymorphie der Kerne. Besonders Fall II fällt auf durch eine allgemeine Verbreiterung der Kerne durch wechselnde Gestalt und durch verschiedene Färbbarkeit. Wesentliche Unterschiede zwischen den polymorphen Kernen der zellreichen, un- 
verdächtigen Myome liessen sich nicht finden, vielmehr handelt es sich lediglich um Wiederholungen der schon dort beobachteten Bilder. In dem letzten Fall, den ich als Myosarkom diagnostiziere, finden sich gleichfalls die beschriebenen Kernveränderungen. Die Kerne dieser Geschwulst sind in ihrem ganzen Verhalten denen von Fall II sehr ähnlich. Jedenfalls zeigen sie keine irgendwie erheblichen Abweichungen von den Kernen, wie sie in den zellreichen, unverdächtigen und sarkomverdächtigen Myomen zu sehen waren. Ob übrigens diese polymorphe Kerne führenden Muskelzellen der von mir beschriebenen, zellreichen Myome und der Myosarkome ausgereifte oder etwa noch unreife Muskelzellen darstellen, wage ich nicht bestimmt $z u$ entscheiden; ich sehe aber keinen Grund, an der Ausreifung der Zellen zu zweifeln. Den einzelnen Zellen ihren Sarkomcharakter anzusehen, halte ich, wenigstens in meinem Fall von Myosarkom, für ganz unmöglich. Ich möchte nochmals hervorheben, dass ich mich von der Bedeutung der Kernveränderungen für die Diagnose des Myosarkoms durch meine Untersuchungen nicht habe überzeugen können.

Einen Wegweiser für die Diagnose des Myosarkoms hoffte ich in dem Vorhandensein von Kernteilungen zu finden. Ich habe daraufhin die vier verdächtigen Geschwülste besonders durchsucht und in der Tat in allen 4 Fällen sichere Kernteilungsfiguren nachweisen können. Von den 10 unverdächtigen Myomen fand ich, wenn auch nach langem Suchen, bei 3 ganz zweifellose Kernteilungsfiguren, bei dem einen davon sogar in verhältnismässig grosser Anzahl. Ich halte es für möglich, dass von den anderen 7 Myomen bei der Durchsuchung weiterer Schnitte sich in dem einen oder anderen noch hätten Kernteilungen finden lassen. Schon nach dem angegebenen Befund glaube ich, dass bei genügend eingehender Untersuchung wohl in jedem Myom Kernteilungsfiguren nachzuweisen sind, vorausgesetzt, dass es lebenswarm fixiert worden ist. Durch experimentelle Untersuchung ist ja festgestellt, dass im frisch exstirpierten Gewebe Kernteilungen, die schon angefangen haben, zu Ende geführt werden, neue Teilungen aber nicht mehr beginnen. Für die Fälle, in denen ich in Myomen keine Kernteilungen nachweisea konnte, dürfen vielleicht äussere Umstände bei der Konservierung der Präparate verantwortlich gemacht werden. Jedenfalls glaube ich, dass das Vorhandensein von Kernteilungen überhaupt nicht zur Unterstützung der Diagnose Myosarkom herangezogen werden darf. 
Dagegen besteht nach meinen Untersuchungen ein entsprechendes Verhältnis zwischen Zahl der Kernteilungen und dem Zellreichtum der Geschwulst, was ja schon a priori anzunehmen ist. Für die Beurteilung der Geschwulst von Bedeutung ist erst das gehäufte Auftreten von Kernteilungsfiguren, wie wir es im oben beschriebenen Myosarkom vor uns haben, wo durchschnittlich 2-3 in einem Gesichtsfeld bei Immersion liegen. Dabei möchte ich auf die Wichtigkeit hinweisen, gerade die jüngsten und noch nicht regressiv-metamorphotisch veränderten Geschwulstpartien aufzusuchen, da natürlich ausschliesslich in diesen die Kernteilungsfiguren auftreten werden.

Das in den Mallory-Ogata-Präparaten studierte Verhalten der interzellulären Fibrillen ergab bei den unverdächtigen Myomen wechselnde Bilder. Bei den verhältnismässig zellärmeren Myomen waren die Fibrillen viel zahlreicher, dünner und länger als bei den zellreicheren Myomen, in denen sie wenige plumpe Stränge bildeten. Dieselben Typen waren bei den verdächtigen Geschwülsten wiederzufinden. Fall I und II zeigten die zahlreichen langen und gestreckt verlaufenden Fibrillen. Fall III und das Myosarkom wiesen einander sehr ähnliche ausgesprochene Bilder des anderen Typs auf. Die beiden letzteren Fälle gleichen sich ferner im Gegensatz zu den anderen untersuchten Geschwülsten durch ihre sehr ausgedehnte hyaline Degeneration. Die Untersuchung der hyalinisierenden Partien in den Geschwülsten ergab für alle Fälle, wo sie überhaupt vorhanden waren, folgenden Nebenbefund. Die hyalinen Partien im Myom resp. muskelzelligen Sarkom entstehen nicht, wie es von einigen Seiten wohl noch angenommen wird, durch Einlagerung homogener Massen in die Spalten des Bindegewebes, sondern das mit Hyalin bezeichnete Gewebe stellt die zusammengesinterten schon ursprünglich vorhandenen Myofibrillen dar, die allerdings zuerst eine Verdickung erfahren. Unter Berücksichtigung dieser meincr Ansicht nach bewiesener Entstehung des Hyalins im Myom halte ich es für möglich, dass das in den Fällen 3 und 4 sehr deutlich ausgesprochene, bei einigen unverdächtigen Myomen schon erkennbare Verhalten der Myotibrillen, nämlich das Vorhandensein weniger, aber dickerer und plumperer Fibrillen, nur ein Ausdruck der beginnenden hyalinen Umwandlung ist. Man müsste sich vorstellen, dass schon in den noch am besten erhaltenen Partien des Tumorgewebes Fibrillen zusammengesintert sind und diese dadurch weniger zahlreich und viel plumper als in 
anderen nicht hyalinisierenden Geschwülsten erscheinen. Vielleicht darf auch die oben erwähnte Beobachtung. Mallory's, der in einem rapid wachsenden zellreichen Uterustumor in manchen Partien gleichzeitig mit unregelmässigen Kernen und mit Kernteilungsfiguren nicht so gut entwickelte Fibrillen sah, in meinem Sinne aufgefasst werden, dass es sich hier um die ersten Anfänge hyaliner Entartung zellreichen Gewebes handelte.

Den geschilderten Befund der Intercellularsubstanz möchte ich gerade mit Berücksichtigung genau des gleichen Verhaltens in Fall III keineswegs charakteristisch für das Myosarkom halten, sondern lediglich für ein Zeichen der beginnenden hyalinen Degeneration.

Eine andere Frage wäre, ob eine hyaline Degeneration nicht vorzugsweise die zellreichen Myome befällt. Man könnte sich vorstellen, dass sehr zellreiche Myome und ebenso Myosarkome so schnell wachsen, dass die Ernährung des Gewebes in verschiedenen Teilen eine nicht genügende wird. Infolge dieser mangelhaften Ernährung gehen die Zellen zu Grunde und die hyalin sich umbildende Grundsubstanz bleibt zurück. Die Betrachtung von allerdings nicht zahlreichen Myomen schien mir darauf hinzuweisen, dass man in zellreichen Myomen häufiger hyaline Entartung findet als in zellarmen. Auch die in Lehrbüchern abgebildeten Präparate von hyaliner Degeneration der Myome zeigen sehr zellreiche Geschwülste (cf. Aschoff II Fig. 425, Schmaus-Herzheimer Fig. 543).

Das Auftreten von Hyalin gerade in den zellreichen Myomen wäre eine Stütze für die von Aschoff geäusserte Ansicht, dass die Hyalinbildung in Myomen als Vernarbungsprozess aufzufassen sei. Auch Krämer stellt sich in seiner Arbeit über die Rückbildungsvorgänge in den Schilddrüsenadenomen auf den Standpunkt, dass die hyaline Rückbildung eine Art Vernarbung darstellt. Dieselbe Auffassung auf die Myome angewandt, bringt eine Arbeit aus jüngster Zeit von Simpson.

Eine besondere Bedeutung für die Diagnose des Myosarkoms scheinen die mehrkernigen Riesenzellen zu besitzen. Allerdings können sie, wenn auch wahrscheinlich nur sehr selten, auch im gewöhnlichen Myom auftreten, wie die von mir in einem sonst durchaus gutartigen Myom gefundene Riesenzelle und die wenigen bei Fall 1 nachweisbaren Riesenzellen zeigen. In grosser Anzahl habe ich sie dagegen nur in dem Myosarkom auftreten 
sehen, bei dem sie sich sowohl ganz besonders häufig an den Grenzpartien gegen die hyalinen Massen, als auch in den jüngeren Partien der Geschwulst fanden. Gerade die letzteren sind wohl für die Beurteilung der Geschwulst von besonderer Bedentung. Dass die Riesenzellen überhaupt für die Diagnose von Wichtigkeit sind, möchte ich besonders betonen, da mit Ausnahme von UlescoStroganowa, sie in der kasuistisehen Literatur des Myosarkoms kaum erwähnt werden.

Besonderen Wert lege ich endlich der Abgrenzung gegen die normale Uterusmuskulatur bei.

Während alle anderen von mir untersuchten Geschwülste überall durchaus scharfe Grenzen zeigten, war bei dem Myosarkom zwar kein eigentliches infiltratives Wuchern, wohl aber ein Einwachsen in die Lymphgefässe zu beobachten.

Das Vorhandensein von Metastasen beseitigt jeden Zweifel an der Diagnose Myosarkom.

Nachdem ich die Ergebnisse meiner histologischen Untersuchungen über zellreiche Myome und Myosarkome besprochen habe, möchte ich darauf eingehen, in wiefern diese für die Erage nach der Häufigkeit des Vorkommens von Sarkom in Myomen verwertet werden können. Wie schon eingangs erwähnt, schwanken darüber die Angaben in der Literatur.

So hat Fehling unter 403 Fibromyomen 8 Sarkome (1,9 pCt.), Hofmeyer unter 578 Fibromyomen 11 Sarkome $(1,9$ pCt.). Winter gelang es unter 753 Fibromyomen 27 Sarkome $(3,5$ pCt.) festzustellen; er weist darauf hin, dass oft nur die mikroskopische Untersuchung möglichst vieler Stellen die sarkomatöse Natur von "Myomen" nachweisen kann und glaubt, dass die Annahme einer sarkomatösen Degeneration in 4 pCt. aller Fälle der Wahrheit am nächsten kommt. Flatau berichtet über 104 Uterusmyome, von denen bei 5 ( $=4,8$ pCt.) eine sarkomatöse Degeneration sich herausstellte. Basso endlich fand bei 105 Bindegewebsgeschwülsten in 5,7 pCt. Sarkome.

Von den neueren Angaben führe ich die von v. Franqué mit 3-6 pCt., und die ron Menge mit $4-5$ pCt. an. Walthard stellte unter 20 schnellwachsenden Tumoren 5 Sarkome fest. Schliesslich berichtet Warnekros in einer soeben erschienenen Arbeit aus der Bumm'schen Klinik, dass er bei 78 exstirpierten Myomen $7 \mathrm{mal}$ sarkomatöse Veränderungen fand; demnach wären 
fast 10 pCt. der zunächst als Myome imponierenden Geschwülste in Wahrheit Myosarkome.

Ich habe in dieser Beziehung das Material des Freiburger Pathologischen Instituts zusammengestellt. Die Durchsicht der Journale ergab, dass in den Jahren 1910 und 1911 im ganzen 544 Präparate, die für eine Untersuchung über Myome in Betracht kamen, dem Institut überwiesen waren. Es waren in der Mehrzahl Uteri, die, gleichgültig aus welcher Indikation, operativ entfernt waren, bei einer kleinen Anzahl davon, die enukleierte Myome darstellte, war nicht der ganze Uterus der pathologisch-anatomischen Untersuchung zugängig gewesen. In einem Falle wurde ein Spindelzellensarkom der Uteruswand ohne gleichzeitiges Vorhandensein von Myom festgestellt. Bei 309 von den 544 exstirpierten Uteri und Myomen wurden Myome festgestellt (= $=56,8 \mathrm{pCt}$.), unter diesen 309 Myomen wurden in 5 Fällen sarkomverdächtige Uterusgeschwülste beobachtet. 4 davon stellen die oben von mir beschriebenen Fälle dar, von denen nur einer als sicheres Myosarkom anerkannt werden konnte, während bei einem zweiten die Diagnose fraglich bleibt. Von dem fünften Fall war nur ein mikroskopisches Präparat aufbewahrt worden, das sich schlecht erhalten hat, so dass ich von einer genaueren Beschreibung absehen muss. Der Vollständigkeit halber möchte ich aber doch den bei der damaligen Untersuchung des Falls im Journal eingetragenen Bericht folgen lassen:

"Bei dem grösseren Myomknoten handelt es sich um ein einfaches Myom, bei einem mehr bräunlich gefärbten Knoten des Tubenwinkels um einen sehr zellreichen, aus kurzen Spindelzellen aufgebauten Tumor, den man als sarkomverdächtig bezeichnen kann. Ein destruierendes Wachstum ist nicht mit Sicherheit nachzuweisen."

Nach dieser Beschreibung sowie auf Grund der Betrachtung des einzigen allerdings schlecht erhaltenen Präparats möchte ich auch diesen Tumor als zellreiches Myom, nicht aber als Sarkom gelten lassen.

Somit bleiben von den 309 Myomen nur der einzige Fall von sicherem Myosarkom und der weitere fragliche Fall übrig.

Ich habe die Zahl der benignen Myome noch erhöhen können, indem ich das Sektionsmaterial des Freiburger Instituts im Jahre 1911 berücksichtigte. Bei 184 weiblichen Leichen wurden 21 Myome gefunden. In einem Falle (unserem Fall IV) wurde Sarkom fest- 
gestellt. Die anderen 20 Myome zeigten auch mikroskopisch keine verdächtige Histologie. Die Diagnose Sarkom kann für diese Fälle ausserdem mit absoluter Sicherheit ausgeschlossen werden, da die vollständige Sektion der Myomträgerinnen keine Metastasen ergeben hat. Es kommt also bei Zusammenrechnung des Materials auf 329 Myome ein sicherer und ein fraglicher Fall von muskelzelligem Sarkom ( $=0,3$ pCt. eventl. 0,6 pCt.).

Das überraschende Resultat dieser Zusammenstellung ist; dass Sarkome nur in sehr seltenen Fällen auftreten. Ich bin überzengt, dass die Werte, die für die Wahrscheinlichkeit einer "sarkomatösen Degeneration von Myomen" angegeben werden, viel zu hoch gegriffen sind. Uebrigens will ich auf die Frage, ob im pathologischanatomischen Sinne überhaupt von einer solchen „Degeneration von Myomen zu Sarkomen" gesprocben werden darf, oder $a b$ es sich bei den als Sarkom festgestellten Tumoren nicht vielmehr um von Anfang an sarkomatöse Geschwülste handelt, nicht eingehen. Es kommt mir lediglich darauf an, zu zeigen, bei wie vielen der als Myom imponierenden Geschwülste ein bindegewebszelliges oder muskelzelliges Sarkom zu finden ist. Ein Bindegewebssarkom, wie es nach der Literatur zweifellos in "Myomen" vorkommt, war in meinem Material nicht zu finden.

Bei dem relativ kleinen Material von 329 Myomen, das meinen Untersuchungen zugrunde liegt, kann ich natürlich nicht allgemein behaupten, dass Sarkom in 0,3 pCt. (oder 0,6 pCt.) aller Fälle von Myom sich findet. Ich glaube aber bestimmt, dass diese Degeneration in weniger als 1 pCt. aller Fälle vorkommt.

Den Widerspruch meiner Schlussergebnisse mit den in der Literatur niedergelegten Angaben glaube ich durch die Unsicherheit der pathologisch-anatomischen Diagnose erklären zu können. Es mögen in der Tat bei der bisherigen Aufstellung von Statistiken über die Häufigkeit der Sarkome Verwechslungen mit zellreichen Myomen vorgekommen sein. Wie aus der Beschreibung der zellreichen Myome und des Myosarkoms hervorgeht, können diese Geschwülste sehr ähnlich aussehen und eine Entscheidung kann sehr schwer sein. Für die Diagnose des Sarkoms möchte ich vor allem den Kernveränderungen und dem Auftreten einzelner Kernteilungsfiguren keinen entscheidenden Wert beimessen, wie das vielleicht bei der bisherigen Beurteilung geschehen ist. Vielmehr sollte die Diagnose Myosarkom des Uterus erst ausgesprochen werden, wenn neben grossem Zellgehalt und den beschriebenen Kernverände- 
rungen gehäufte Kernteilungsfiguren und Riesenzellen vorkommen oder wenn die Geschwulst in ihrem Verhalten zum angrenzenden Gewebe oder in Metastasenbildung einen zweifellos malignen Charakter zeigt.

Muskelgeschwülste des Uterus, die Metastasen setzen, möchte ich im Anschluss an Robert Meyer nicht maligne Myome, sondern mauskelzellige Sarkome resp. Myosarkome nennen. Einmal scheint auch mir eine mehr zufällige Metastasenbildung unwahrscheinlich, dann aber zeigen die in der Literatur beschriebenen Fälle von "malignem Myom" auch histologische Besonderheiten, so dass das Vorkommen von klinisch malignen, histologisch benignen Myomen einstweilen als unbewiesen gelten muss.

Für sehr wohl möglich halte ich, dass es nicht in allen Fällen möglich ist, aus dem histologischen Bilde eine sichere Entscheidung zwischen der Diagnose zellreiches Myom oder Myosarkom zu treffen. Diese Fälle können nur nach dem weiteren klinischen Verlauf und dem Ergebnis der späteren Sektion beurteilt werden.

\section{Erklärıng der Abbildung auf Tafel XI.}

Figur 9. Fall IV. Hyaline Degeneration des Geschwulstgewebes in der Umgebung eines Gefässes. Die rotgefärbten Kerne und grösseren Riesenzellkerne, zum Teil pyknotisch, rücken in Reihen zusammen, während die blauen interzellulären Fibrillen zu breiten Bändern zusammensintern und schliesslich die blangefärbten hyalinen Massen bilden, die aber immer noch in ihrer Struktur ihre Entstehung ans zusammengerückten Fibrillen und Fibrillenbündeln zeigen. 


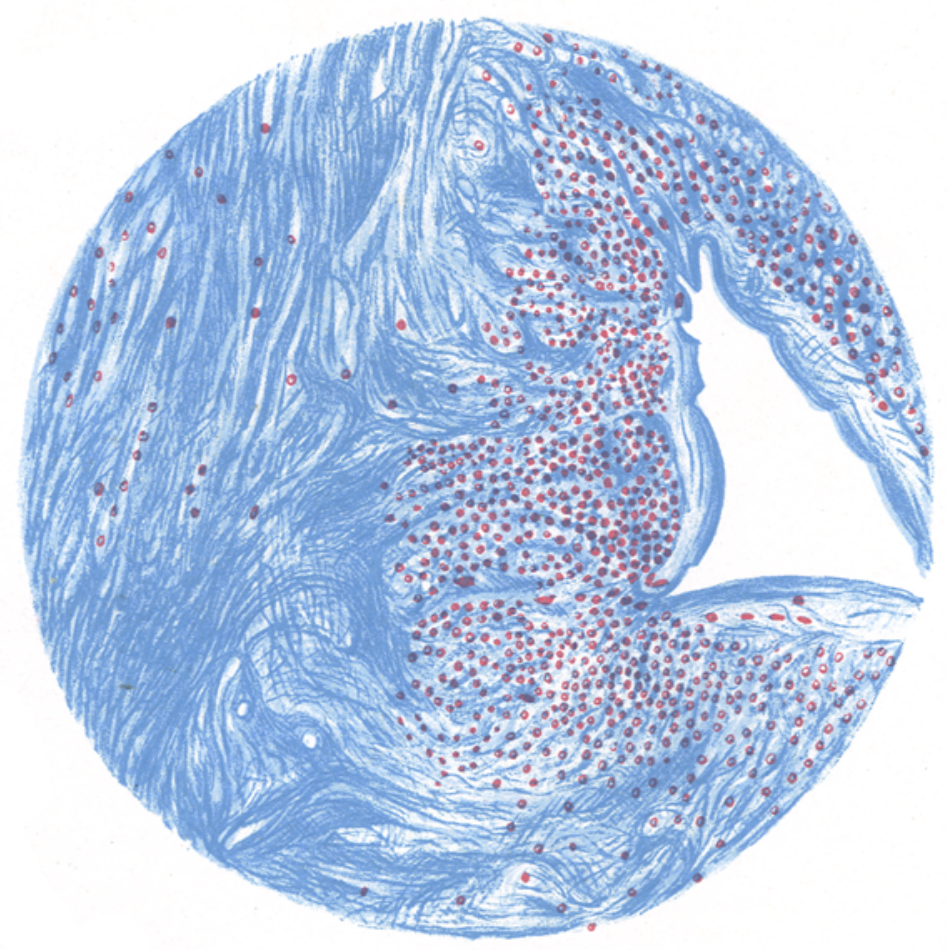

Raab, Zellreiche Myome und Myosarkome des Vterus. 